Draft Version March 22, 2021

Typeset using LATEX twocolumn style in AASTeX63

\title{
The influence of metallicity on the Leavitt Law from geometrical distances of Milky Way and Magellanic Clouds Cepheids
}

\author{
Louise Breuval, ${ }^{1}$ Pierre Kervella, ${ }^{1}$ Piotr Wielgórski, ${ }^{2}$ Wolfgang Gieren, ${ }^{3}$ Dariusz Graczyk, ${ }^{4}$ Boris Trahin, ${ }^{1}$ \\ Grzegorz Pietrzyński, ${ }^{2}$ Frédéric Arenou, ${ }^{5}$ Behnam Javanmardi, ${ }^{1}$ And Barteomiej Zgirski ${ }^{2}$ \\ ${ }^{1}$ LESIA, Observatoire de Paris, Université PSL, CNRS, Sorbonne Université, Université de Paris, 5 place Jules Janssen, 92195 Meudon, \\ France \\ ${ }^{2}$ Nicolaus Copernicus Astronomical Centre, Polish Academy of Sciences, Bartycka 18, 00-716 Warszawa, Poland \\ ${ }^{3}$ Universidad de Concepción, Departamento de Astronomía, Casilla 160-C, Concepción, Chile \\ ${ }^{4}$ Centrum Astronomiczne im. Mikotaja Kopernika, Polish Academy of Sciences, Rabiańska 8, 87-100, Toruń, Poland \\ ${ }^{5}$ GEPI, Observatoire de Paris, Université PSL, CNRS, 5 place Jules Janssen, 92190 Meudon, France
}

(Received 29 January 2021; Revised 12 March 2021; Accepted 18 March 2021)

Submitted to ApJ

\begin{abstract}
The Cepheid Period-Luminosity (PL) relation is the key tool for measuring astronomical distances and for establishing the extragalactic distance scale. In particular, the local value of the Hubble constant $\left(H_{0}\right)$ strongly depends on Cepheid distance measurements. The recent Gaia Data Releases and other parallax measurements from the Hubble Space Telescope (HST) already enabled to improve the accuracy of the slope $(\alpha)$ and intercept $(\beta)$ of the PL relation. However, the dependence of this law on metallicity is still largely debated. In this paper, we combine three samples of Cepheids in the Milky Way (MW), the Large Magellanic Cloud (LMC) and the Small Magellanic Cloud (SMC) in order to derive the metallicity term (hereafter $\gamma$ ) of the PL relation. The recent publication of extremely precise LMC and SMC distances based on late-type detached eclipsing binary systems (DEBs) provides a solid anchor for the Magellanic Clouds. In the MW, we adopt Cepheid parallaxes from the early third Gaia Data Release. We derive the metallicity effect in $V, I, J, H, K_{S}, W_{V I}$ and $W_{J K}$. In the $K_{S}$ band we report a metallicity effect of $-0.221 \pm 0.051 \mathrm{mag} / \mathrm{dex}$, the negative sign meaning that more metal-rich Cepheids are intrinsically brighter than their more metal-poor counterparts of the same pulsation period.
\end{abstract}

Keywords: parallaxes - stars: distances - stars: variables: Cepheids - distance scale - metallicity

\section{INTRODUCTION}

The Cepheid Period-Luminosity (PL) relation, discovered by Henrietta Leavitt (Leavitt \& Pickering 1912) about a century ago, is an essential tool for measuring astronomical distances since it represents the first rung of the extragalactic distance ladder. This law is used to measure distances to type Ia supernovae (SNe Ia) host galaxies, and thus plays a key role in the determination of the Hubble constant $\left(H_{0}\right)$. This parameter currently exhibits a tension of at least $\sim 4 \sigma$ between its measurement in the early Universe by Planck Collaboration et al.

louise.breuval@obspm.fr
(2020) assuming a $\Lambda$-CDM cosmology and the local estimate based on Cepheid distances (Riess et al. 2021). The precise calibration of the PL relation is therefore of paramount importance to reach a $1 \%$ determination of the Hubble constant.

While the slope $(\alpha)$ and intercept $(\beta)$ of the Leavitt law are generally consistent between various studies, the value and even the sign of the metallicity term $(\gamma$, defined as $M=\alpha \log P+\beta+\gamma[\mathrm{Fe} / \mathrm{H}])$ are still debated and constitute $0.5 \%$ of the error budget of $H_{0}$ (Riess et al. 2016). Some empirical studies report a metallicity dependence consistent with $\gamma \sim 0 \mathrm{mag} / \mathrm{dex}$ : Udalski et al. (2001) concludes with a null-effect from the study of a metal-poor galaxy in optical bands, Storm et al. (2011a) finds a null effect in all bands except in 
$W_{V I}$ and Wielgórski et al. (2017) derive a gamma value consistent with zero in optical and NIR bands. Still, a large majority of the analysis investigating the metallicity effect derived a negative sign, with values ranging between -0.2 and $-0.5 \mathrm{mag} / \mathrm{dex}$ (Freedman \& Madore 1990; Macri et al. 2006; Saha et al. 2006; Gieren et al. 2018; Groenewegen 2018). This trend would indicate that metal-rich Cepheids are brighter than metal-poor ones. However, the study by Romaniello et al. (2008) yielded a metallicity effect of the opposite sign, confirming the theoretical predictions (Caputo et al. 2000; Bono et al. 2008; Fiorentino et al. 2013).

In this paper, we aim at determining the effect of metallicity on the PL relation by combining samples of Cepheids in the Milky Way (MW) and in the Magellanic Clouds (MCs), taking advantage of the large range of metallicity covered by the Cepheids in these 3 galaxies (from +0.08 dex to $-0.75 \mathrm{dex}$ ). Most of the Cepheids located in distant galaxies hosting SNIa have metallicities within this range, therefore our results are directly applicable to extragalactic studies of the distance scale (e.g. Javanmardi et al. 2021).

Recently, Pietrzyński et al. (2019) and Graczyk et al. (2020) measured the most precise distances to date for the Large Magellanic Cloud (LMC) and Small Magellanic Cloud (SMC) respectively, based on enhanced samples of late-type detached eclipsing binaries (DEBs). These distances allow us to obtain a precise calibration of the PL relation in the LMC and SMC. For Milky Way (MW) Cepheids, we use the early third Gaia Data Release (EDR3) which recently provided parallaxes of unprecedented precision for hundreds of galactic Cepheids.

In Sect. 2, we present our samples of Cepheids in the three galaxies and in Sect. 3 we provide the distances we adopted for each sample. Then in Sect. 4 we estimate the metallicity effect by fitting the Period-LuminosityMetallicity (PLZ) relation in the three galaxies. We discuss the results in Sect. 5 .

\section{SAMPLES OF CEPHEIDS}

\section{1. $M W$ Cepheids}

We gather a sample of Milky Way Cepheids for which well-covered light curves are available. In the NIR $J, H$ and $K$ bands, we combine the catalogs by Welch et al. (1984), Laney \& Stobie (1992), Barnes et al. (1997) and Monson \& Pierce (2011). The data from these four studies are found to be in close agreement, with residuals of $0.013,0.010$ and $0.002 \mathrm{mag}$ in $J, H$ and $K$ respectively (Monson \& Pierce 2011). We adopt these values as photometric zero-point uncertainties for the NIR photometry. Additional NIR data were also found in Feast et al. (2008), we consider that including this source of data does not impact the homogeneity and the dispersion of the data since it only affects four stars of the sample. In the optical $V$ and $I$ bands, we use the catalog from Berdnikov (2008) that provides photometry in the Johnson-Cousins system for a large number of Cepheids. Since it is a compilation of data from various catalogs by the same author, we adopt a photometric zero-point uncertainty of $0.010 \mathrm{mag}$.

For each star and in each filter, we phase the data at the date of maximum luminosity and we obtain intensity-averaged mean apparent magnitudes by performing light curve fitting using Fourier series. Depending on the properties of the different light curves (such as the presence of bumps, steep variations, or to prevent the introduction of unphysical oscillations when the data are too dispersed or not dense enough), we adapt the number of Fourier modes, and thus of free parameters, in order to obtain a satisfactory representation of the light curve. A Fourier decomposition of order three is generally sufficient for an usual Cepheid light curve such as $\delta$ Cep, and is up to order six for a more complex star such as RS Pup. We derived the statistical uncertainties on the mean magnitudes from the scatter of each light curve. In some few cases, a very large number of data points are available $(>300)$ and result in unrealistic small errors: in these cases we adopt a minimum error of $0.006 \mathrm{mag}$.

For long period Cepheids, large phase shifts may degrade the quality of the fit, the photometry being spread over four decades. Therefore, period changes were taken into account for the phasing of long period stars such as SV Vul, GY Sge or RS Pup (Kervella et al. 2017). We adopted a polynomial model of up to degree five for the pulsation period.

We carefully analyse the light curves: we exclude Cepheids for which less than 8 data-points are available (MW Cepheids have on average 35 data points in NIR and 160 in optical) and Cepheids that have poor quality photometry or insufficient phase coverage. Finally, we convert all the NIR data in the 2MASS system using the transformations from Monson \& Pierce (2011). The systematics related to these transformations are negligible. Examples of a well covered light curve and of a poor-quality light curve are provided in Fig. 8 and 9 in Appendix.

We select Cepheids pulsating in the fundamental mode according to the reclassification by Ripepi et al. (2019). For stars that were not available in this catalog, we adopted by order of priority the pulsation modes from Groenewegen (2018), from the Variable Star indeX (VSX, Watson et al. 2006) and from Luck (2018). 

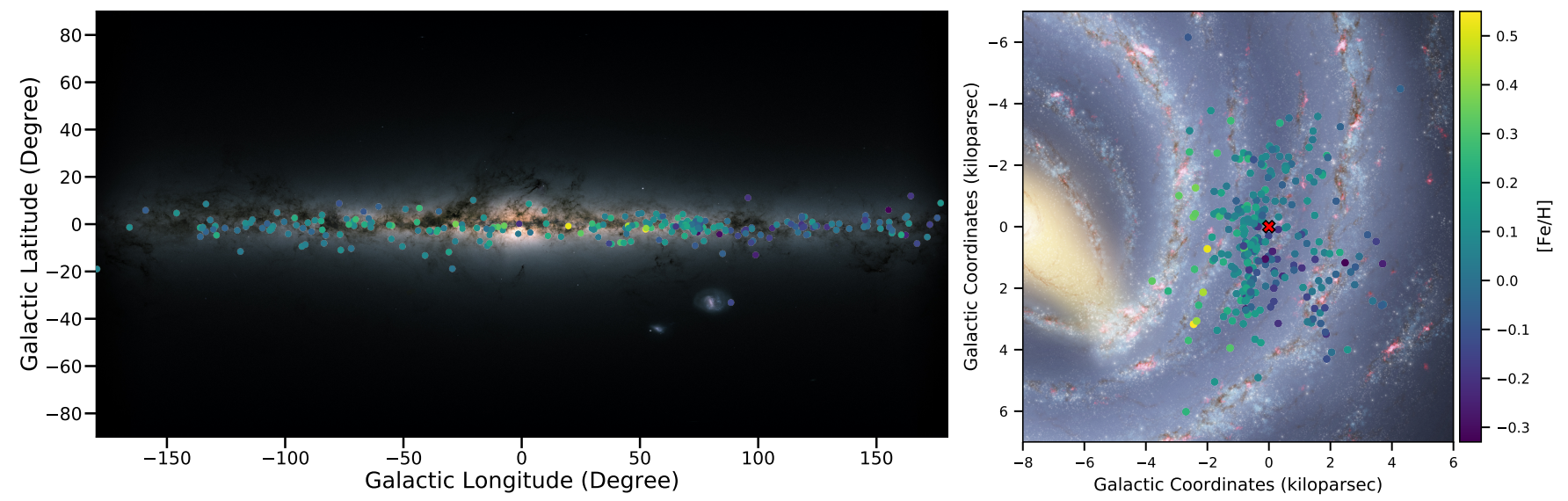

Figure 1. Galactic maps projected on the sky (left) and on the galactic plane (right) showing the distribution of the MW Cepheid sample. The color scale represents the metallicity $[\mathrm{Fe} / \mathrm{H}]$ and the red cross is the position of the Solar System.

We adopt reddening values from Fernie et al. (1995) with a 0.94 scaling factor as suggested by Groenewegen (2018), and from Acharova et al. (2012) if not available in the latter. We adopt an uncertainty of 0.05 if it is not provided.

For MW Cepheids, we search for individual metallicities in Genovali et al. (2015). This catalog provides mean abundances based on high resolution spectra for 75 Cepheids. For stars that are not available in this catalog, we adopt the values from Genovali et al. (2014): they provide homogeneous Cepheid metallicities from their group and compiled from the literature, rescaled to their solar abundance. The individual metallicities are represented in Fig. 1 by colored points, they range from -0.33 dex to +0.55 dex. The gradient of metallicity in the MW is particularly visible, with metalrich Cepheids located closer to the galactic center than metal-poor ones. These individual metallicities have a weighted mean value of $0.083 \pm 0.019$ dex with a scatter of 0.14 dex. In the following, we adopt this weighted mean value for all MW Cepheids for consistency and homogenity with the LMC and SMC samples that only have a mean metallicity, but also because the current precision of the individual metallicities is not sufficient for a thorough calibration of the metallicity effect.

The Cepheids of our MW sample are represented in Fig. 1 and their main parameters are listed in Table 4 and Table 5 in Appendix.

\subsection{LMC Cepheids}

We build a sample of LMC Cepheids by combining the OGLE-IV photometry in $V$ and $I$ bands (Soszyński et al. 2015) with the multi-epoch observations from the LMC Near-Infrared Synoptic Survey by Macri et al. (2015) taken with the CPAPIR camera on the $1.5 \mathrm{~m}$ CTIO telescope. We update their NIR mean magnitudes to bring them into better agreement with the 2MASS system using the following relations (L. Macri, priv. comm.). These were derived by matching $\sim 34000$ stars in common between their Table A1 and the 2MASS Point Source Catalog (Cutri et al. 2003), with $12<H<13.5$, $K>11.5$ and $-0.5<J-K<1.4$ mag:

$$
\begin{aligned}
J_{2 \mathrm{MASS}}= & J_{\mathrm{M} 15}-0.0167+0.0205\left(J_{\mathrm{M} 15}-K_{\mathrm{M} 15}-0.4\right) \\
& +0.0101\left(J_{\mathrm{M} 15}-K_{\mathrm{M} 15}-0.4\right)^{2} \\
H_{2 \mathrm{MASS}}= & H_{\mathrm{M} 15}+0.0116-0.0054\left(J_{\mathrm{M} 15}-K_{\mathrm{M} 15}-0.4\right) \\
& -0.0189\left(J_{\mathrm{M} 15}-K_{\mathrm{M} 15}-0.4\right)^{2} \\
K_{2 \mathrm{MASS}}= & K_{\mathrm{M} 15}+0.0162+0.0227\left(J_{\mathrm{M} 15}-K_{\mathrm{M} 15}-0.4\right) \\
& -0.0595\left(J_{\mathrm{M} 15}-K_{\mathrm{M} 15}-0.4\right)^{2}
\end{aligned}
$$

We adopt a photometric zero-point uncertainty of 0.02 mag in all bands. Since some Cepheids exhibit large brightness variations during a pulsation cycle, we consider that single-epoch photometry is not precise enough to derive reliable mean magnitudes, therefore we discarded the mean magnitudes derived by Inno et al. (2016) from template fitting on 2MASS single-point data and IRSF measurements.

We perform a quality check on this initial sample: we reject stars with magnitude uncertainties larger than $1 \%$ and with less than 5 data points (LMC Cepheids have on average 43 data points in NIR and 147 in optical), and we only consider fundamental mode Cepheids. We reject Cepheids located outside a radius of 3 degrees around the LMC center in order to avoid outliers such as stars that do not belong to the LMC or that are strongly affected by its geometrical effects (see Sect. 3.2). We adopt reddening values from the Górski et al. (2020) reddening map. The final sample of LMC Cepheids contains 1446 stars in the $V$ band and 807 stars in $K_{S}$, it is listed in Table 6 in Appendix and provided as supplementary material online. A map of the final sample of LMC Cepheids is represented in Fig. 2. For 


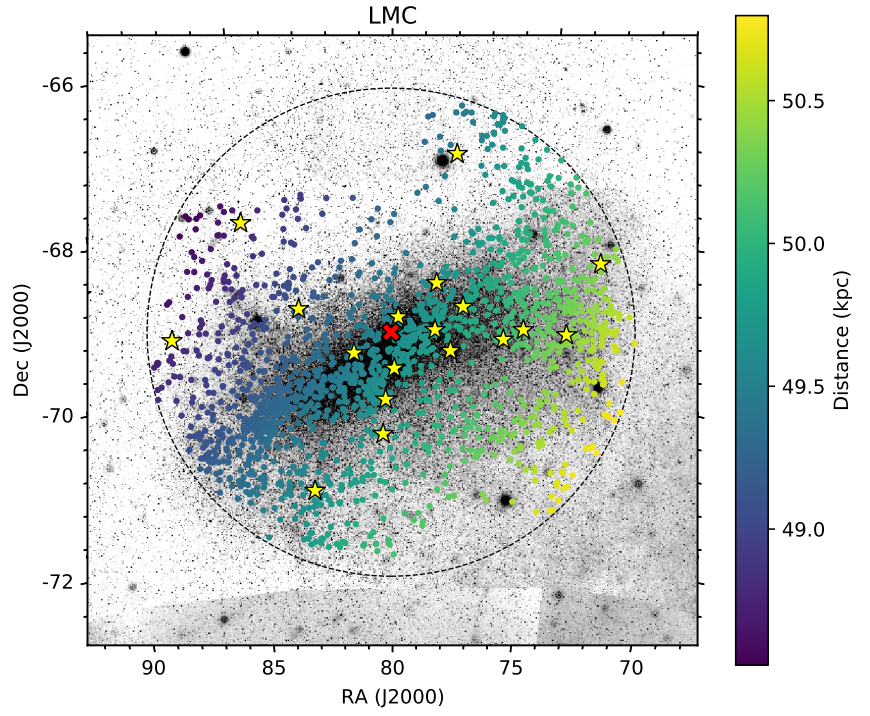

Figure 2. Map of the LMC Cepheids considered in our study. Yellow stars are the eclipsing binaries from Pietrzyński et al. (2019) and the red cross is the center of the LMC. The dashed circle represents a radius of 3 degrees around the LMC center.

LMC Cepheids, we adopt the mean metallicity used by Gieren et al. (2018), that compiles several estimates from various studies: $[\mathrm{Fe} / \mathrm{H}]_{\mathrm{LMC}}=-0.34 \pm 0.06$ dex. The uncertainties take into account the homogenization of the different measurements.

\subsection{SMC Cepheids}

We assemble a sample of SMC Cepheids by taking the mean magnitudes from the VISTA survey of the Magellanic Clouds (VMC) (Ripepi et al. 2016) crossmatched with OGLE IV photometry by Soszyński et al. (2015). Unfortunately, we do not have $H$-band photometry for SMC Cepheids because we rejected data from single epoch photometry and template fitting. Results in the $H$ band are therefore derived from the combination of MW and LMC Cepheids only. Magnitudes in the VISTA system were converted into the 2MASS system using the equations from Ripepi et al. (2016):

$$
\begin{aligned}
& J^{\prime}=J_{\mathrm{VMC}}+0.070\left(J_{\mathrm{VMC}}-K_{\mathrm{VMC}}\right) \\
& K^{\prime}=K_{\mathrm{VMC}}-0.011\left(J_{\mathrm{VMC}}-K_{\mathrm{VMC}}\right)
\end{aligned}
$$

We perform an additional correction (L. Macri, priv. comm.) derived by matching $\sim 7000$ stars in common between the VMC DR4 and the 2MASS Point Source Catalog, with $J>12.25, K>11.5$ and $-0.5<J-K<$ 1.4 mag:

$$
\begin{aligned}
& J_{2 \mathrm{MASS}}=J^{\prime}-0.0087-0.0010\left(J^{\prime}-K^{\prime}-0.4\right) \\
& K_{2 \mathrm{MASS}}=K^{\prime}+0.0011-0.0087\left(J^{\prime}-K^{\prime}-0.4\right)
\end{aligned}
$$

We adopt a photometric zero-point uncertainty of 0.02 mag for all bands. As we did for the LMC sample, we also reject SMC Cepheids with magnitude uncertainties larger than $1 \%$, with less than 5 data points (SMC Cepheids have on average 17 data points in NIR and 46 in optical) and we only keep Cepheids pulsating in the fundamental mode. As for the LMC sample, we adopt reddening values from the Górski et al. (2020) reddening map.

While the LMC has a rather simple geometry, the SMC is very elongated along the line of sight: we select Cepheids located in a region of $0.6 \mathrm{deg}$ around the SMC center, which covers an area of $1.3 \mathrm{kpc}$ width. Since the SMC distance is derived from detached eclipsing binaries (DEBs), this selection ensures that the Cepheids are located in the same region as these DEBs. The final SMC sample has 284 stars in the $V$ band and 295 stars in $K_{S}$, it is listed in Table 7 in Appendix and provided as supplementary material. A map of our final sample of SMC Cepheids is represented in Fig. 3.

For SMC Cepheids, we adopt the mean metallicity used by Gieren et al. (2018), that compiles several estimates from various studies: $[\mathrm{Fe} / \mathrm{H}]_{\mathrm{SMC}}=$ $-0.75 \pm 0.05$ dex. Similar to the LMC value, the uncertainty takes into account the homogenization of the different measurements.

\section{DISTANCES}

In order to calibrate the Leavitt law, one needs to derive the absolute magnitude of each Cepheid from its apparent luminosity and from its distance.

\subsection{Distances to Milky Way Cepheids}

Recently, the early third Gaia Data Release provided new parallaxes for Milky Way Cepheids (Gaia Collaboration 2020). We perform a first quality check of Gaia EDR3 parallaxes based on the Renormalised Unit Weight Error (RUWE) provided in the catalog. This parameter reflects the quality of the parallax of a star compared to other stars of the same color and brightness. Its value is expected to be close to 1 for wellbehaved sources (Lindegren et al. 2020a). In particular, the RUWE is sensitive to the photocentric motion of unresolved objects, therefore it can be used to detect possible astrometric binaries. We discard the Cepheids of our sample that have a RUWE $>1.4$ : this selection corresponds to approximately $13 \%$ of our MW sample and removes the stars that are possibly affected by saturation or contamination by a bright neighbour companion. In particular, all the outliers noticed by eye on 


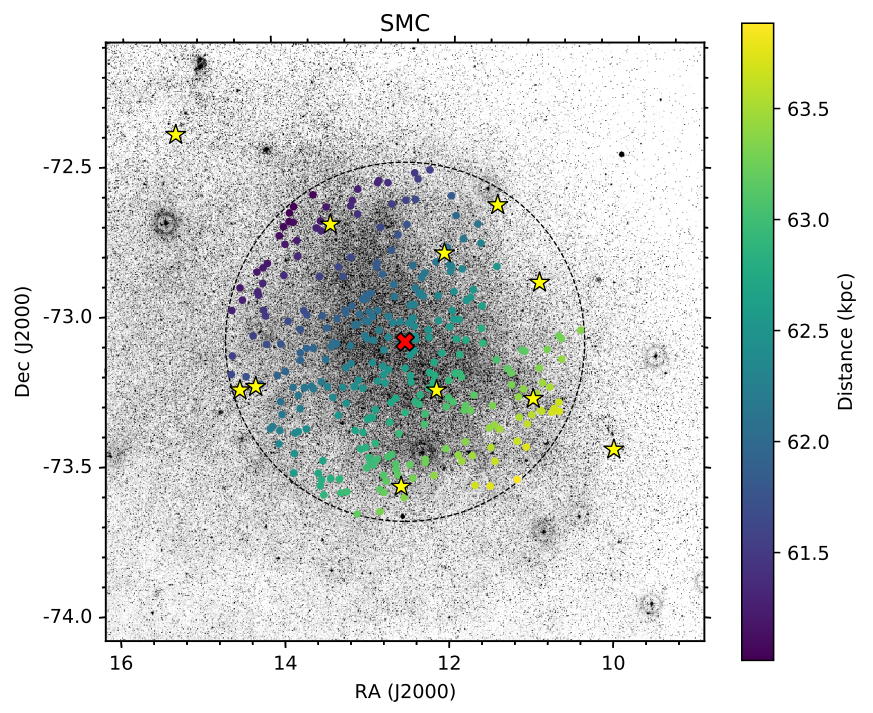

Figure 3. Map of the SMC Cepheids considered in our study. Yellow stars are the eclipsing binaries from Graczyk et al. (2020) and the red cross is the center of the SMC. The dashed circle represents a radius of 0.6 degree around the SMC center.

the PL relation are affected by a large RUWE, therefore the quality check based on this parameter appears to be relevant for our purpose.

Riess et al. (2021) use a different indicator: they identify stars with a goodness of fit (GOF) larger than 12.5 as having a compromised parallax. We find the GOF and the RUWE selections to have a very similar effect on our sample: adopting this GOF criteria for the quality check instead of the RUWE leads to rejecting exactly the same stars, except T Mon, V0496 Aql and VW Pup, that have a RUWE of $1.72,1.56$ and 1.41, and a GOF of $12.11,10.90$ and 11.36 respectively. The RUWE criterion seems slightly more selective than the GOF limit adopted by Riess et al. (2021). Adopting a threshold of RUWE $<1.4$ corresponds to a limit GOF of 10 .

One method to check if a Cepheid is an astrometric binary is to look for proper motion anomalies between the observations by Hipparcos and Gaia. Using the approach described in Kervella et al. (2019), we find 20 Cepheids with a high proper motion anomaly signal. However, none of them were identified by their high RUWE or GOF and they do not appear as outliers, therefore we do not exclude them.

Cepheids are variable stars and therefore their brightness and colour can change significantly during a pulsation cycle. This effect was not taken into account in the processing of Gaia DR2 astrometry and resulted in additional systematics, noise and dispersion for variable stars parallaxes (Breuval et al. 2020). The correction for this chromatic effect on Cepheid parallaxes is still absent from Gaia EDR3 (Lindegren et al. 2020a). However, the number of observations obtained for each star increased consequently between Gaia DR2 ( $\sim 22$ months) and Gaia EDR3 ( 34 months). We assume in this paper that the noise induced by this effect is negligible for Gaia EDR3 parallaxes.

For each Cepheid we correct for the parallax zeropoint (ZP) by using the Python tool ${ }^{1}$ described in Lindegren et al. (2020a). This ZP correction takes into account the ecliptic latitude, magnitude and colour of each star. Our MW Cepheids cover a range of magnitudes from $G=3$ to $G=12 \mathrm{mag}$. For our sample of MW Cepheids, we find the ZP to vary between -4 and $-54 \mu$ as with a median value of $-27 \mu$ as $(\sigma=10 \mu \mathrm{as})$, which is very similar to the median parallax offset derived by Riess et al. (2021). Following Lindegren et al. (2020a) who recommend to include an uncertainty of a few micro arcseconds in the ZP, we adopt a systematic error of $5 \mu$ as on this quantity. Considering our sample of Cepheids, this error is equivalent to an average systematic uncertainty of $0.020 \mathrm{mag}$ in distance modulus. In Sect. 5.1, we discuss the influence of adopting this individual ZP correction compared with the uniform ZP of $-17 \mu$ as derived from quasars.

We find 13 Cepheids to fall in the range between $G=$ 10.8 and $G=11.2 \mathrm{mag}$, where a transition of window classes occurs (Fig. 1 in Lindegren et al. 2020b). In this particular range, the value of the parallax zero-point can possibly be affected so we quadratically add $10 \mu$ as to the parallax uncertainty.

Finally, we increase all Gaia EDR3 parallax uncertainties by $10 \%$, following Riess et al. (2021) to account for potential excess uncertainty. This correction has significantly reduced since Gaia DR2, where it was recommended to increase parallax uncertainties by $30 \%$.

\subsection{Distances to LMC Cepheids}

Recently, Pietrzyński et al. (2019) estimated the distance to the LMC with a $1 \%$ precision based on detached-eclipsing binaries (DEBs): $d_{\mathrm{LMC}}=49.59 \pm$ 0.09 (stat.) \pm 0.54 (syst.) kpc. This method for measuring distances is independent from Cepheids and relies on surface-brightness relations, established by precise interferometric measurements. We use this value as initial distance to our Cepheids and we add a corrective term depending on the position of each Cepheid in the LMC, assuming the disc geometry derived by OGLE from Cepheids by Jacyszyn-Dobrzeniecka et al. (2016). First

\footnotetext{
${ }^{1}$ https://www.cosmos.esa.int/web/gaia/edr3-code
} 
we compute the cartesian coordinates $\left(x_{i}, y_{i}, z_{i}\right)$ of each Cepheid from their equatorial coordinates $\left(\alpha_{\mathrm{i}}, \delta_{\mathrm{i}}\right)$ using the transformations:

$$
\left\{\begin{aligned}
x_{i} & =-d_{\mathrm{LMC}} \cos \delta_{i} \sin \left(\alpha_{\mathrm{i}}-\alpha_{\mathrm{LMC}}\right) \\
y_{i}= & d_{\mathrm{LMC}}\left[\sin \delta_{i} \cos \delta_{\mathrm{LMC}}\right. \\
& \left.\quad-\cos \delta_{i} \sin \delta_{\mathrm{LMC}} \cos \left(\alpha_{\mathrm{i}}-\alpha_{\mathrm{LMC}}\right)\right] \\
z_{i}= & c_{1} x_{i}+c_{2} y_{i}+d_{\mathrm{LMC}}
\end{aligned}\right.
$$

where $\left(\alpha_{\mathrm{LMC}}, \delta_{\mathrm{LMC}}\right)=(80.05,-69.30)$ deg are the coordinates of the LMC center and the coefficients $\left(c_{1}, c_{2}\right)$ $=(0.395 \pm 0.014,-0.215 \pm 0.013)$ are from JacyszynDobrzeniecka et al. (2016). The corrected distance of each LMC Cepheid is:

$$
d_{i}=\sqrt{x_{i}^{2}+y_{i}^{2}+z_{i}^{2}}
$$

The distances of each LMC Cepheid derived with this correction are located in a range of $\pm 1.5 \mathrm{kpc}$ around the mean LMC distance from Pietrzyński et al. (2019). They are represented by the colors in Fig. 2 .

\subsection{Distances to SMC Cepheids}

The distance to the SMC was recently measured by Graczyk et al. (2020) with a precision of $1.5 \%$ using the same method as used in Pietrzyński et al. (2019) for the LMC: from a sample of 15 DEBs, a distance of $d_{\mathrm{SMC}}=62.44 \pm 0.47$ (stat.) \pm 0.81 (syst.) $\mathrm{kpc}$ is derived. However, the SMC has a large extension along the line of sight (Subramanian \& Subramaniam 2012; Jacyszyn-Dobrzeniecka et al. 2016; Ripepi et al. 2017), which makes the distance to its core region particularly difficult to measure, contrary to the LMC that has a rather simple geometry. In this section, we take into account the SMC elongated shape in order to derive corrected distances to each of its Cepheids. For each SMC Cepheid of coordinates $\left(\alpha_{\mathrm{i}}, \delta_{\mathrm{i}}\right)$, we compute the cartesian coordinates $\left(x_{i}, y_{i}\right)$ such that:

$$
\left\{\begin{array}{l}
x_{i}=-d_{\mathrm{SMC}} \cos \delta_{i} \sin \left(\alpha_{\mathrm{i}}-\alpha_{\mathrm{SMC}}\right) \\
y_{i}=d_{\mathrm{SMC}}\left[\sin \delta_{i} \cos \delta_{\mathrm{SMC}}\right. \\
\left.\quad-\cos \delta_{i} \sin \delta_{\mathrm{SMC}} \cos \left(\alpha_{\mathrm{i}}-\alpha_{\mathrm{SMC}}\right)\right]
\end{array}\right.
$$

where $\left(\alpha_{\mathrm{SMC}}, \delta_{\mathrm{SMC}}\right)=(12.54,-73.11) \operatorname{deg}($ Ripepi et al. 2017). Then we used the equations corresponding to the blue lines in Fig. 4 of Graczyk et al. (2020):

$$
\left\{\begin{array}{l}
d_{i}(x)=(3.086 \pm 0.066) x_{i}+d_{\mathrm{SMC}} \\
d_{i}(y)=(-3.248 \pm 0.118) y_{i}+d_{\mathrm{SMC}}
\end{array}\right.
$$

We adopt the mean value of $d_{i}(x)$ and $d_{i}(y)$ as the final distance of each Cepheid. The elongated shape of

\begin{tabular}{|c|c|c|c|c|}
\hline Band & $\alpha$ & $\beta$ & $\sigma$ & $N^{(*)}$ \\
\hline \multicolumn{5}{|c|}{ MW $(a)$} \\
\hline$V$ & $-2.443 \pm 0.031$ & $-3.296 \pm 0.024$ & 0.25 & 178 \\
\hline$I$ & $-2.780 \pm 0.028$ & $-3.981 \pm 0.024$ & 0.23 & 150 \\
\hline$W_{V I}$ & $-3.289 \pm 0.026$ & $-5.030 \pm 0.025$ & 0.21 & 149 \\
\hline$J$ & $-3.050 \pm 0.029$ & $-4.498 \pm 0.026$ & 0.18 & 97 \\
\hline$H$ & $-3.160 \pm 0.028$ & $-4.762 \pm 0.024$ & 0.17 & 97 \\
\hline$K_{S}$ & $-3.207 \pm 0.028$ & $-4.848 \pm 0.022$ & 0.17 & 97 \\
\hline$W_{J K}$ & $-3.317 \pm 0.028$ & $-5.086 \pm 0.026$ & 0.17 & 97 \\
\hline \multicolumn{5}{|c|}{$\mathrm{LMC}^{(b)}$} \\
\hline$V$ & $-2.704 \pm 0.007$ & $-3.284 \pm 0.033$ & 0.23 & 1446 \\
\hline$I$ & $-2.916 \pm 0.005$ & $-3.910 \pm 0.033$ & 0.15 & 1460 \\
\hline$W_{V I}$ & $-3.281 \pm 0.008$ & $-4.877 \pm 0.038$ & 0.08 & 1432 \\
\hline$J$ & $-3.127 \pm 0.005$ & $-4.385 \pm 0.033$ & 0.12 & 805 \\
\hline$H$ & $-3.160 \pm 0.005$ & $-4.696 \pm 0.033$ & 0.11 & 808 \\
\hline$K_{S}$ & $-3.217 \pm 0.005$ & $-4.737 \pm 0.033$ & 0.10 & 807 \\
\hline$W_{J K}$ & $-3.272 \pm 0.008$ & $-4.974 \pm 0.039$ & 0.10 & 806 \\
\hline \multicolumn{5}{|c|}{$\mathrm{SMC}^{(c)}$} \\
\hline$V$ & $-2.594 \pm 0.012$ & $-3.196 \pm 0.038$ & 0.28 & 284 \\
\hline$I$ & $-2.871 \pm 0.008$ & $-3.841 \pm 0.038$ & 0.22 & 297 \\
\hline$W_{V I}$ & $-3.334 \pm 0.014$ & $-4.834 \pm 0.043$ & 0.12 & 283 \\
\hline$J$ & $-2.956 \pm 0.004$ & $-4.317 \pm 0.038$ & 0.17 & 294 \\
\hline$H$ & - & - & - & - \\
\hline$K_{S}$ & $-3.163 \pm 0.002$ & $-4.670 \pm 0.038$ & 0.15 & 295 \\
\hline$W_{J K}$ & $-3.326 \pm 0.002$ & $-4.916 \pm 0.043$ & 0.14 & 295 \\
\hline
\end{tabular}

Table 1. Results of the PL fit of the form $M=\alpha(\log P-$ $0.7)+\beta$ in the Milky Way, the Large Magellanic Cloud and the Small Magellanic Cloud.

Note- $\left(^{*}\right)$ The number of stars is given after the sigma clipping procedure and the period cuts.

(a) Mean $[\mathrm{Fe} / \mathrm{H}]=+0.083 \pm 0.019 \mathrm{dex}$

(b) Mean $[\mathrm{Fe} / \mathrm{H}]=-0.34 \pm 0.06 \mathrm{dex}$

(c) Mean $[\mathrm{Fe} / \mathrm{H}]=-0.75 \pm 0.05$ dex

the SMC is highlighted by the dispersion of the derived distances between $+5 \mathrm{kpc}$ and $-6 \mathrm{kpc}$ around the mean value $d_{\mathrm{SMC}}$, which represents almost $10 \%$ of the mean value. The distances of our sample of SMC Cepheids are represented on the map in Fig. 3. A discussion about the elongated shape of the SMC and its impact on our results is provided in Sect. 5.2. 


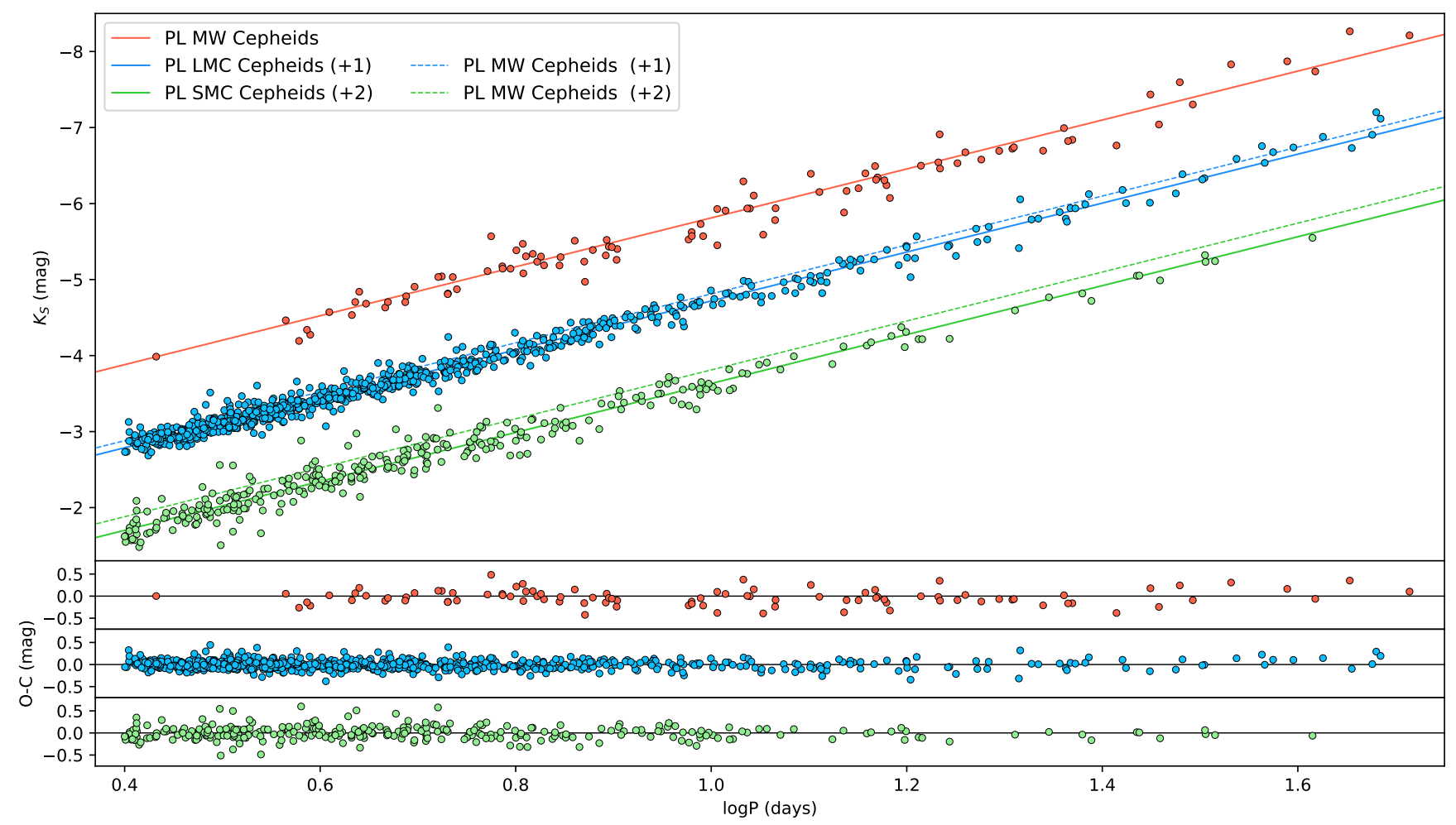

Figure 4. Fit of the PL relation in $K_{S}$ for MW, LMC and SMC Cepheids. The lower panel shows the residual between the Cepheid absolute magnitudes and the corresponding PL fit for each of the three galaxies. The LMC and SMC relations were offset by +1 and +2 mag, respectively, for visualization purposes.

\section{THE METALLICITY EFFECT FROM MILKY WAY AND MAGELLANIC CLOUD CEPHEIDS}

In this section, we aim at estimating the metallicity term $\gamma$ of the Leavitt law. In Sect. 4.1, we start by fitting the $\alpha$ and $\beta$ coefficients of the PL relation in each of the three galaxies, without considering the metallicity term. In Sect. 4.2, we include the metallicity for each galaxy and derive the third term of the PLZ relation by combining the three galaxies.

\subsection{The Period-Luminosity relation}

We adopt the Cepheid samples described in Sect. 2. In a first place, we correct apparent magnitudes for the extinction by adopting the reddening law from Cardelli et al. (1989) and O'Donnell (1994) assuming $R_{V}=3.135$ which yields $A_{\lambda}=R_{\lambda} E(B-V)$ with $R_{I}=1.894, R_{J}=$ $0.892, R_{H}=0.553$ and $R_{K_{S}}=0.363$. We also derive optical and NIR Wesenheit indices (Madore 1982) as defined by $W_{V I}=I-1.526(V-I)$ and $W_{J K}=K_{S}-$ $0.686\left(J-K_{S}\right)$. Wesenheit magnitudes are particularly convenient for calibrating the PL relation since they are independent of reddening.

We account for the width of the instability strip by adding quadratically to the photometry uncertainties the intrinsic scatter in each band: this quantity is obtained by subtracting quadratically the measurement errors (photometric inhomogeneities, differential extinction, geometrical effects, phase corrections, etc) from the scatter of the PL relation: we adopt a width of the instability strip of $0.07 \mathrm{mag}$ in NIR bands $\left(J, H, K_{S}\right.$ and $W_{J K}$ ) from Persson et al. (2004), $0.15 \mathrm{mag}$ in $V$ and $0.09 \mathrm{mag}$ in $I$ from Macri et al. (2006) and finally 0.08 mag in $W_{V I}$ from Madore et al. (2017). We derive the absolute magnitude $M_{\lambda}$ of each Cepheid from their distance $d$ (in $\mathrm{kpc}$ ) and dereddened apparent magnitude $m_{\lambda}$ :

$$
M_{\lambda}=m_{\lambda}-5 \log d-10
$$

In the Milky Way, the distance is obtained at the first order by taking the inverse of the parallax. In order to avoid biases due to this inversion, we adopt the approach introduced by Feast \& Catchpole (1997) and Arenou \& Luri (1999), consisting in fitting the Astrometric Based Luminosity (ABL) function instead of absolute magnitudes:

$$
\mathrm{ABL}=\pi_{(\mathrm{mas})} 10^{0.2 \mathrm{~m}_{\lambda}-2}=10^{\mathrm{M}_{\lambda} / 5}
$$

where:

$$
M_{\lambda}=\alpha_{\lambda}\left(\log P-\log P_{0}\right)+\beta_{\lambda}
$$



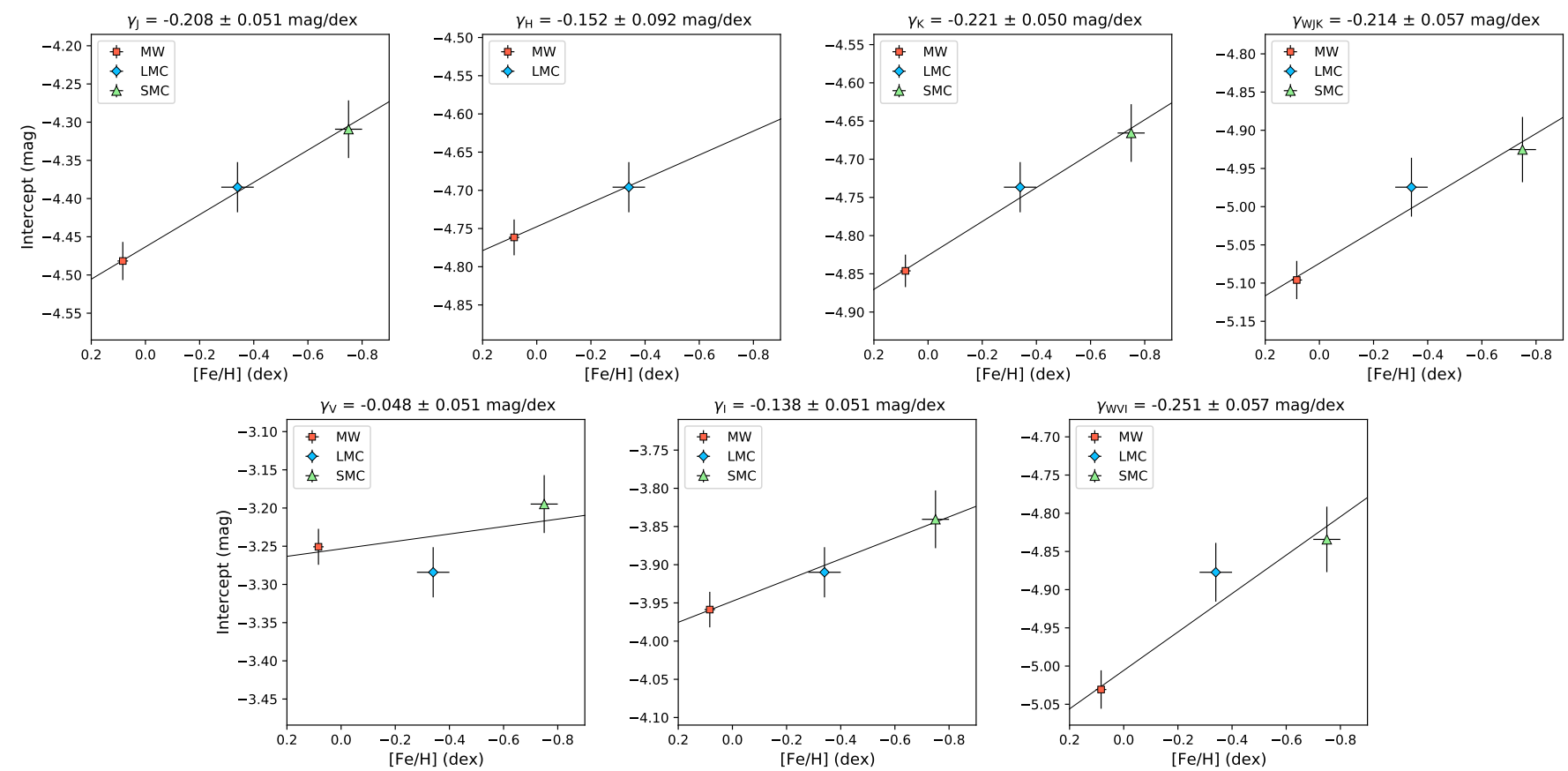

Figure 5. Intercept of the PL relation represented as a function of metallicity in $J, H, K, V, I$, and Wesenheit bands.

We adopt a pivot period of $\log P_{0}=0.7$ which represents the median period of our Cepheid sample. This approach ensures minimum correlations between the fitted coefficients. We perform a $3 \sigma$ clipping procedure on the PL relation to remove possible outliers.

A non-linearity in the SMC PL relation was highlighted at the short-periods end $(\log P<0.4)$ (EROS Collaboration et al. 1999). For LMC and SMC Cepheids, Chown et al. (2021) detect a break in the PL relation at $\log P=0.29$ and also at very long periods $(\log P=1.72)$. Cepheids beyond these limits are found to deviate from the global PL fit and can affect both the slope and the zero-point. Additionally, the shortperiod edge of the PL relation is potentially affected by first-overtone contamination. In the following, we exclude all Cepheids with periods shorter than 2.5 days $(\log P=0.4)$ and longer than 52 days $(\log P=1.72)$. Finally, we include the systematics on the LMC and SMC distance moduli (respectively $0.026 \mathrm{mag}$ and 0.032 $\mathrm{mag}$ ) and the photometric zero-points provided in Sect. 2 on the intercept error. We use the curve_fit function from the scipy Python library in a Monte Carlo algorithm to derive the PL coefficients and the 16th and 84th percentiles of the distribution to derive the uncertainties. The PL relations derived for each galaxy are provided in Table 1, where both the slope and intercept are fitted.

In each band, the intercept increases with decreasing metallicity, i.e. it becomes less negative from the MW to the LMC and in turn to the SMC. In the NIR, the intercept changes by $\sim 0.18$ mag between the MW and the SMC, possibly indicating a strong dependence with metallicity. We note that our $K_{S}$ band calibration in the $\mathrm{MW}$ is in good agreement with the result by Breuval et al. (2020) based on Gaia DR2 parallaxes. The fit of the PL relation in the $K_{S}$ band performed in each of the three galaxies is represented in Fig. 4.

\subsection{The Period-Luminosity-Metallicity relation}

In this section, we now calibrate the dependence of the PL intercept $\beta$ with metallicity. First, we fit the PL relation of the form $M=\alpha(\log P-0.7)+\beta$ in each of the three galaxies separately with a common slope fixed to the LMC value. As in previous section, the systematics due to the LMC and SMC distance and to the photometric zero-point are included in quadrature to the intercept random error. The intercept $\beta$ contains the metallicity term such that:

$$
\beta=\gamma[\mathrm{Fe} / \mathrm{H}]+\delta
$$

In Fig. 5 are represented the intercepts of the PL relations in the MW, LMC and SMC as a function of metallicity. We fit Eq. 4 with a Monte Carlo algorithm to derive the $\gamma$ and $\delta$ coefficients, and we adopt the 16th and 84th percentiles of the distribution to derive the random errors. A histogram representing the distribution of the $\gamma$ values obtained with the Monte Carlo algorithm is represented in Fig. 6.

The results of the fit are listed in Table 2. In the NIR, we report a strong metallicity effect of $-0.208 \pm$ 


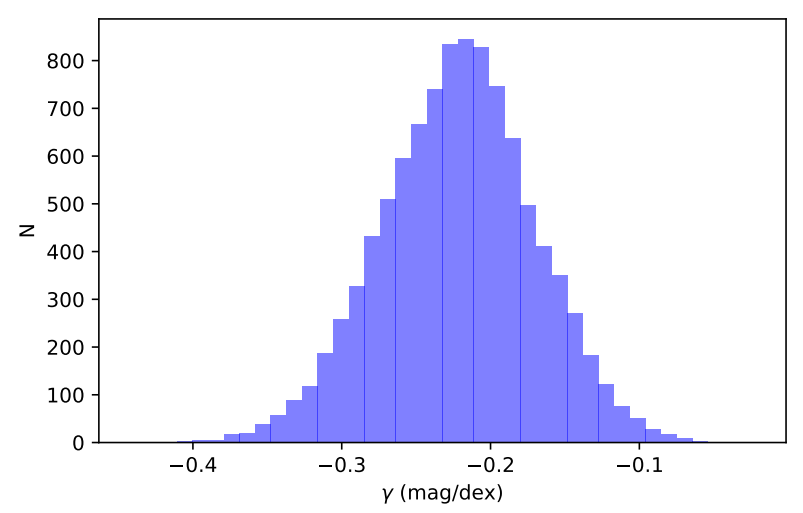

Figure 6. Histogram of the $\gamma$ values obtained in the $K_{S}$ band by the Monte Carlo algorithm iterated 10000 times.

$0.051 \mathrm{mag} / \mathrm{dex}$ in $J,-0.152 \pm 0.092 \mathrm{mag} / \mathrm{dex}$ in $H$ and $-0.221 \pm 0.050 \mathrm{mag} / \mathrm{dex}$ in $K_{S}$. The NIR Wesenheit index $W_{J K}$ shows a similar dependence with $-0.214 \pm 0.057 \mathrm{mag} / \mathrm{dex}$. These results agree by $1 \sigma$ with Gieren et al. (2018), who used the Infrared Surface Brightness Technique (Fouqué \& Gieren 1997; Storm et al. 2011b) to derive the distances to the Cepheids in their MW, LMC and SMC samples, an approach different and independent from the one used in the present study. In the NIR Wesenheit index $W_{H}$, Riess et al. (2019) find an effect of $-0.170 \pm 0.060 \mathrm{mag} / \mathrm{dex}$, which is also close to our results in the NIR. In optical bands, we derive a weaker effect than in the NIR with $-0.048 \pm 0.051 \mathrm{mag} / \mathrm{dex}$ in $V$ and of $-0.138 \pm 0.051$ $\mathrm{mag} / \mathrm{dex}$ in $I$. These values also agree at $1 \sigma$ with Gieren et al. (2018), and the value in $V$ is also consistent at $1 \sigma$ with the differential study of LMC and SMC PL relations by Wielgórski et al. (2017). On average, our results are located between the values by Wielgórski et al. (2017), consistent with a null metallicity effect, and the work by Gieren et al. (2018) that derive a strong negative effect. In the $H$ band, we derive a metallicity effect weaker than in other NIR bands, likely because it is derived from the MW and LMC samples only (due to the lack of $H$-band photometry for SMC Cepheids). We conclude with the general trend being that the sensitivity to metallicity increases in absolute sense and becomes more negative from optical to NIR wavelengths. This trend is particularly visible in Fig. 7 .

We note that the PL slope was fixed to the LMC value because this sample contains significantly more stars than the two other ones. However, if the slope is fixed to the value found in the Milky Way or in the SMC, the intercepts agree by $0.2 \%$ in NIR and by $1.4 \%$ in optical. Similarly, the $\gamma$ values agree at $0.2 \sigma$ and 0.8
Table 2. Final results of the PLZ fit of the form $M=$ $\alpha(\log P-0.7)+\delta+\gamma[\mathrm{Fe} / \mathrm{H}]$ and associated uncertainties.

\begin{tabular}{l|cc|cc|cc}
\hline \hline Band & $\alpha$ & $\sigma$ & $\delta$ & $\sigma$ & $\gamma$ & $\sigma$ \\
\hline$V$ & -2.704 & 0.007 & -3.252 & 0.020 & -0.048 & 0.055 \\
$I$ & -2.916 & 0.005 & -3.948 & 0.020 & -0.138 & 0.053 \\
$W_{V I}$ & -3.281 & 0.008 & -5.005 & 0.022 & -0.251 & 0.057 \\
$J$ & -3.127 & 0.005 & -4.463 & 0.022 & -0.208 & 0.052 \\
$H$ & -3.160 & 0.005 & -4.748 & 0.020 & -0.152 & 0.092 \\
$K_{S}$ & -3.217 & 0.004 & -4.826 & 0.019 & -0.221 & 0.051 \\
$W_{J K}$ & -3.273 & 0.008 & -5.075 & 0.022 & -0.214 & 0.057 \\
\hline
\end{tabular}

Note-The uncertainties include the systematics discussed in Sect. 5.2.

$\sigma$ in NIR and optical respectively.

\section{DISCUSSION}

The metallicity term of the PL relation can be sensitive to many different effects. In this section we study the stability $\gamma$ after varying some parameters.

\subsection{Influence of Gaia EDR3 parallax zero-point}

In Sect. 3.1, we corrected each Gaia EDR3 parallax for their individual zero-point by using the Python tool described in Lindegren et al. (2020a). However, in Lindegren et al. (2020b), a uniform parallax zero-point of $-17 \mu$ as is derived from quasars. The results of the PLZ fit obtained after adopting this uniform zero-point are provided in the second part of Table 3 in appendix. They are consistent at the $1 \sigma$ level with the values derived using the individual zero-point, although it gives a slightly more negative metallicity effect in each band. For example, in $K_{S}$ we obtain $\gamma=-0.271 \pm 0.051 \mathrm{mag} / \mathrm{dex}$ compared with $\gamma=-0.221 \pm 0.050 \mathrm{mag} / \mathrm{dex}$ with individual zero-points. This effect can be explained by the individual zero-points being on average more negative than $-17 \mu$ as for our sample of MW Cepheids.

We also investigate whether the individual zero-point correction by Lindegren et al. (2020a) is adapted to the most distant Cepheids: we remove from our sample the Cepheids with a parallax smaller than 0.3 mas and derive the PL relation in $K_{S}$ without these stars. Using this PL relation, we compute the expected parallax of the most distant Cepheids and compare it with the Gaia EDR3 parallax corrected by the individual zeropoint. We find a good agreement between the predicted parallaxes and the values from Gaia EDR3 with the Lindegren et al. (2020a) individual correction. From this study, we confirm that the individual zero-point 


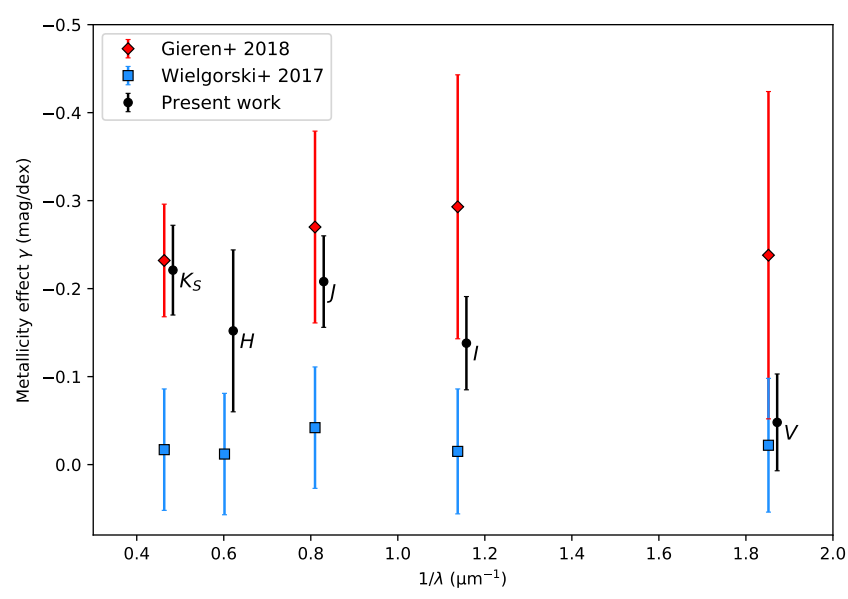

Figure 7. Metallicity effect as a function of wavelength, compared with values from the literature. The error bars include the systematics discussed in Sect. 5.2. For visualization purposes, the $\mathrm{X}$ axis was slightly shifted for our values so that the error bars do not overlap, but they correspond to the same wavelength as the literature values.

correction from Lindegren et al. (2020a) is adapted to the most distant MW Cepheids of our sample.

\subsection{Influence of the SMC sample}

As mentionned in Sect. 2.3 and 3.3, the distance to the core region of the SMC is particularly difficult to measure. From their sample of DEBs, Graczyk et al. (2020) derive an uncertainty of about $2 \%$ for the distance to the SMC core region. These DEB systems are unevenly distributed in the central region of the galaxy and their individual distances show a large dispersion around the mean value, ranging from $57 \mathrm{kpc}$ to $67 \mathrm{kpc}$ (see their Fig. 3), which corresponds to $\sim 16 \%$ of the SMC distance. In order to avoid including Cepheids located too far away from the SMC center, we restricted our sample to a region of radius 0.6 degree around the SMC center. With a smaller radius, the contribution of the SMC sample in the PLZ fit becomes smaller than the MW contribution, therefore we consider that the number of retained SMC Cepheids is insufficient. On the other hand, if we assume a radius larger than 0.6 deg around the SMC center, the number of outlier stars increases and the distance of some Cepheids may not correspond to the distance of the SMC core region. In order to test the validity of our hypothesis, we perform the same PLZ fit with a radius of 0.5 and $0.7 \mathrm{deg}$ around the SMC center and report the coefficients in Table 3.

After extending the SMC sample to a radius of 0.7 degree around the galaxy centrer, we find $\gamma$ values in very good agreement (better than $1 \sigma$ ) with the values derived in the initial conditions. When the radius is reduced to 0.5 degree, the metallicity effect still agrees at $1 \sigma$ with the initial conditions in all bands. Considering a smaller region around the SMC center results in a slightly stronger (i.e. more negative) metallicity effect. These results highlight the sensitivity of the metallicity effect with respect to the adopted SMC sample, and in particular to the spatial distribution of the Cepheids considered. Moreover, it emphasizes the necessity to correct each Cepheid distance according to their position in the SMC plane, as we did in Sect. 3.3.

We consider the variation of $\gamma$ within a region of $0.5^{\circ}<R<0.7^{\circ}$ around the SMC center as an additional source of systematic uncertainties: this source of error is at the level of $0.02 \mathrm{mag} / \mathrm{dex}$ in optical bands and of $0.01 \mathrm{mag} / \mathrm{dex}$ in NIR (see Table 3). We adopt the same additional source of uncertainty for the intercept $\delta$, although the latter coefficient is particularly stable when the radius around the SMC center is changed. These systematics are included in the results presented in Table 2.

\section{CONCLUSIONS}

We build large samples of Cepheids in the Milky Way and in the Magellanic Clouds and make use of the most recent and precise distances available to estimate the metallicity effect on the Cepheid PL relation. In the $K_{S}$ band we derive an effect of $\gamma=-0.221 \pm 0.051 \mathrm{mag} / \mathrm{dex}$, in agreement with the value found by Gieren et al. (2018) but more precise. In the $V$ band we derive a weaker effect of $\gamma=-0.048 \pm 0.055 \mathrm{mag} / \mathrm{dex}$, which is consistent with both Wielgórski et al. (2017) and Gieren et al. (2018) within the error bars. We conclude with a nonzero dependence of Cepheid magnitude with metallicity and we confirm its negative sign: metal-rich Cepheids are brighter than metal-poor ones.

The improved precision reached in this work was made possible thanks to the high quality of Gaia EDR3 parallaxes and to the new distances of the two Magellanic Clouds obtained by the Araucaria Project. Combining Milky Way and Magellanic Cloud Cepheids also allows to reach a better precision than previous studies based on Magellanic Clouds only, by the larger range of metallicities they cover. A refined analysis of each light curve ensures the use of accurate mean magnitudes. However, the elongated shape of the SMC in the line of sight remains a source of systematic uncertainty in our study, despite continuous efforts to improve our knowledge of its structure. In this study, we assumed a linear dependence of the PL relation with metallicity, but it might as well be non-linear (Gieren et al. 2018). Additional high resolution spectroscopic metallicity mea- 
surements of both Milky Way and Magellanic Cloud Cepheids should be carried out in the future to even better constrain the metallicity effect, particularly in the NIR, in our effort to further reduce the systematic uncertainty on the determination of the Hubble constant from the Cepheid-SN Ia method.

\section{ACKNOWLEDGMENTS}

We thank the anonymous referee for the very constructive comments that helped us to improve the manuscript. We are grateful to Lucas Macri for providing the photometric transformations. The research leading to these results has received funding from the European Research Council (ERC) under the European Union's Horizon 2020 research and innovation programme under grant agreement No 695099 (project CepBin). This work has made use of data from the European Space Agency (ESA) mission Gaia (http://www.cosmos.esa.int/gaia), processed by the Gaia Data Processing and Analysis Consortium (DPAC, http://www.cosmos.esa.int/web/gaia/dpac/consortium). Funding for the DPAC has been provided by national institutions, in particular the institutions participating in the Gaia Multilateral Agreement. The authors acknowledge the support of the French Agence Nationale de la Recherche (ANR), under grant ANR-15-CE310012-01 (project UnlockCepheids). W.G. and G. P. gratefully acknowledge financial support for this work from the BASAL Centro de Astrofisica y Tecnologias Afines (CATA) AFB- 170002. W.G. and D. G. acknowledge financial support from the Millenium Institute of Astrophysics (MAS) of the Iniciativa Cientifica Milenio del Ministerio de Economia, Fomento y Turismo de Chile, project IC120009. This research made use of Astropy7, a community-developed core Python package for Astronomy (Astropy Collaboration et al. 2018). Support from the Polish National Science Centre grants MAESTRO UMO-2017/26/A/ST9/00446 and from the IdPII 2015000264 and DIR/WK/2018/12 grants of the Polish Ministry of Science and Higher Education is also acknowledged. We used the SIMBAD and VIZIER databases and catalog access tool at the CDS, Strasbourg (France), and NASA's Astrophysics Data System Bibliographic Services. 


\section{REFERENCES}

Acharova, I. A., Mishurov, Y. N., \& Kovtyukh, V. V. 2012, MNRAS, 420, 1590

Arenou, F., \& Luri, X. 1999, in Astronomical Society of the Pacific Conference Series, Vol. 167, Harmonizing Cosmic Distance Scales in a Post-HIPPARCOS Era, ed. D. Egret \& A. Heck, 13-32

Astropy Collaboration, Price-Whelan, A. M., Sipőcz, B. M., et al. 2018, AJ, 156, 123

Barnes, T. G., I., Fernley, J. A., Frueh, M. L., et al. 1997, Publications of the Astronomical Society of the Pacific, 109, 645

Berdnikov, L. N. 2008, VizieR Online Data Catalog, 2285, 0

Bono, G., Caputo, F., Fiorentino, G., Marconi, M., \& Musella, I. 2008, ApJ, 684, 102

Breuval, L., Kervella, P., Anderson, R. I., et al. 2020, A\&A, 643, A115

Caputo, F., Marconi, M., Musella, I., \& Santolamazza, P. 2000, A\&A, 359, 1059

Cardelli, J. A., Clayton, G. C., \& Mathis, J. S. 1989, ApJ, 345,245

Chown, A. H., Scowcroft, V., \& Wuyts, S. 2021, MNRAS, 500,817

Cutri, R. M., Skrutskie, M. F., van Dyk, S., et al. 2003, 2MASS All Sky Catalog of point sources.

EROS Collaboration, Bauer, F., Afonso, C., et al. 1999, A\&A, 348, 175

Feast, M. W., \& Catchpole, R. M. 1997, MNRAS, 286, L1, doi: 10.1093/mnras/286.1.L1

Feast, M. W., Laney, C. D., Kinman, T. D., van Leeuwen, F., \& Whitelock, P. A. 2008, MNRAS, 386, 2115, doi: 10.1111/j.1365-2966.2008.13181.x

Fernie, J. D., Evans, N. R., Beattie, B., \& Seager, S. 1995, Information Bulletin on Variable Stars, 4148

Fiorentino, G., Musella, I., \& Marconi, M. 2013, MNRAS, 434, 2866

Fouqué, P., \& Gieren, W. P. 1997, A\&A, 320, 799

Freedman, W. L., \& Madore, B. F. 1990, ApJ, 365, 186

Gaia Collaboration. 2020, VizieR Online Data Catalog, $\mathrm{I} / 350$

Genovali, K., Lemasle, B., Bono, G., et al. 2013, A\&A, 554, A132

-. 2014, A\&A, 566, A37, doi: 10.1051/0004-6361/201323198

Genovali, K., Lemasle, B., da Silva, R., et al. 2015, A\&A, 580, A17

Gieren, W., Storm, J., Konorski, P., et al. 2018, A\&A, 620, A99, doi: 10.1051/0004-6361/201833263

Górski, M., Zgirski, B., Pietrzyński, G., et al. 2020, ApJ, 889,179
Graczyk, D., Pietrzynski, G., Thompson, I. B., et al. 2020, arXiv e-prints, arXiv:2010.08754

Groenewegen, M. A. T. 2018, A\&A, 619, A8, doi: 10.1051/0004-6361/201833478

Inno, L., Bono, G., Matsunaga, N., et al. 2016, ApJ, 832, 176

Jacyszyn-Dobrzeniecka, A. M., Skowron, D. M., Mróz, P., et al. 2016, AcA, 66, 149

Javanmardi, B., Mérand, A., Kervella, P., et al. 2021, arXiv e-prints, arXiv:2102.12489

Kervella, P., Arenou, F., Mignard, F., \& Thévenin, F. 2019, A\&A, 623, A72, doi: 10.1051/0004-6361/201834371

Kervella, P., Trahin, B., Bond, H. E., et al. 2017, A\&A, 600, A127, doi: 10.1051/0004-6361/201630202

Laney, C. D., \& Stobie, R. S. 1992, A\&AS, 93, 93

Leavitt, H. S., \& Pickering, E. C. 1912, Harvard College Observatory Circular, 173, 1

Lemasle, B., François, P., Bono, G., et al. 2007, A\&A, 467, 283

Lindegren, L., Klioner, S. A., Hernández, J., et al. 2020a, arXiv e-prints, arXiv:2012.03380

Lindegren, L., Bastian, U., Biermann, M., et al. 2020b, arXiv e-prints, arXiv:2012.01742

Luck, R. E. 2018, AJ, 156, 171, doi: $10.3847 / 1538-3881 /$ aadcac

Luck, R. E., Andrievsky, S. M., Kovtyukh, V. V., Gieren, W., \& Graczyk, D. 2011, AJ, 142, 51

Luck, R. E., \& Lambert, D. L. 2011, AJ, 142, 136

Macri, L. M., Ngeow, C.-C., Kanbur, S. M., Mahzooni, S., \& Smitka, M. T. 2015, AJ, 149, 117

Macri, L. M., Stanek, K. Z., Bersier, D., Greenhill, L. J., \& Reid, M. J. 2006, ApJ, 652, 1133

Madore, B. F. 1982, ApJ, 253, 575

Madore, B. F., Freedman, W. L., \& Moak, S. 2017, ApJ, 842,42

Monson, A. J., \& Pierce, M. J. 2011, ApJS, 193, 12, doi: 10.1088/0067-0049/193/1/12

O’Donnell, J. E. 1994, ApJ, 422, 158

Pedicelli, S., Lemasle, B., Groenewegen, M., et al. 2010, A\&A, 518, A11

Persson, S. E., Madore, B. F., Krzemiński, W., et al. 2004, AJ, 128, 2239

Pietrzyński, G., Graczyk, D., Gallenne, A., et al. 2019, Nature, 567, 200

Planck Collaboration, Aghanim, N., Akrami, Y., et al. 2020, A\&A, 641, A6, doi: 10.1051/0004-6361/201833910

Riess, A. G., Casertano, S., Yuan, W., et al. 2021, ApJL, 908, L6 
Riess, A. G., Casertano, S., Yuan, W., Macri, L. M., \& Scolnic, D. 2019, ApJ, 876, 85

Riess, A. G., Macri, L. M., Hoffmann, S. L., et al. 2016, ApJ, 826, 56, doi: 10.3847/0004-637X/826/1/56

Ripepi, V., Molinaro, R., Musella, I., et al. 2019, A\&A, 625, A14

Ripepi, V., Marconi, M., Moretti, M. I., et al. 2016, ApJS, 224, 21

Ripepi, V., Cioni, M.-R. L., Moretti, M. I., et al. 2017, MNRAS, 472, 808

Romaniello, M., Primas, F., Mottini, M., et al. 2008, A\&A, 488, 731

Saha, A., Thim, F., Tammann, G. A., Reindl, B., \& Sand age, A. 2006, ApJS, 165, 108

Soszyński, I., Udalski, A., Szymański, M. K., et al. 2015, AcA, 65, 297. https://arxiv.org/abs/1601.01318

Storm, J., Gieren, W., Fouqué, P., et al. 2011a, A\&A, 534, A95
-. 2011b, A\&A, 534, A94

Subramanian, S., \& Subramaniam, A. 2012, ApJ, 744, 128

Sziládi, K., Vinkó, J., Poretti, E., Szabados, L., \& Kun, M. 2007, A\&A, 473, 579

Udalski, A., Wyrzykowski, L., Pietrzynski, G., et al. 2001, AcA, 51, 221

Watson, C. L., Henden, A. A., \& Price, A. 2006, Society for Astronomical Sciences Annual Symposium, 25, 47

Welch, D. L., Wieland, F., McAlary, C. W., et al. 1984, ApJS, 54, 547, doi: 10.1086/190943

Wielgórski, P., Pietrzyński, G., Gieren, W., et al. 2017, ApJ, 842, 116

Yong, D., Carney, B. W., Teixera de Almeida, M. L., \& Pohl, B. L. 2006, AJ, 131, 2256 


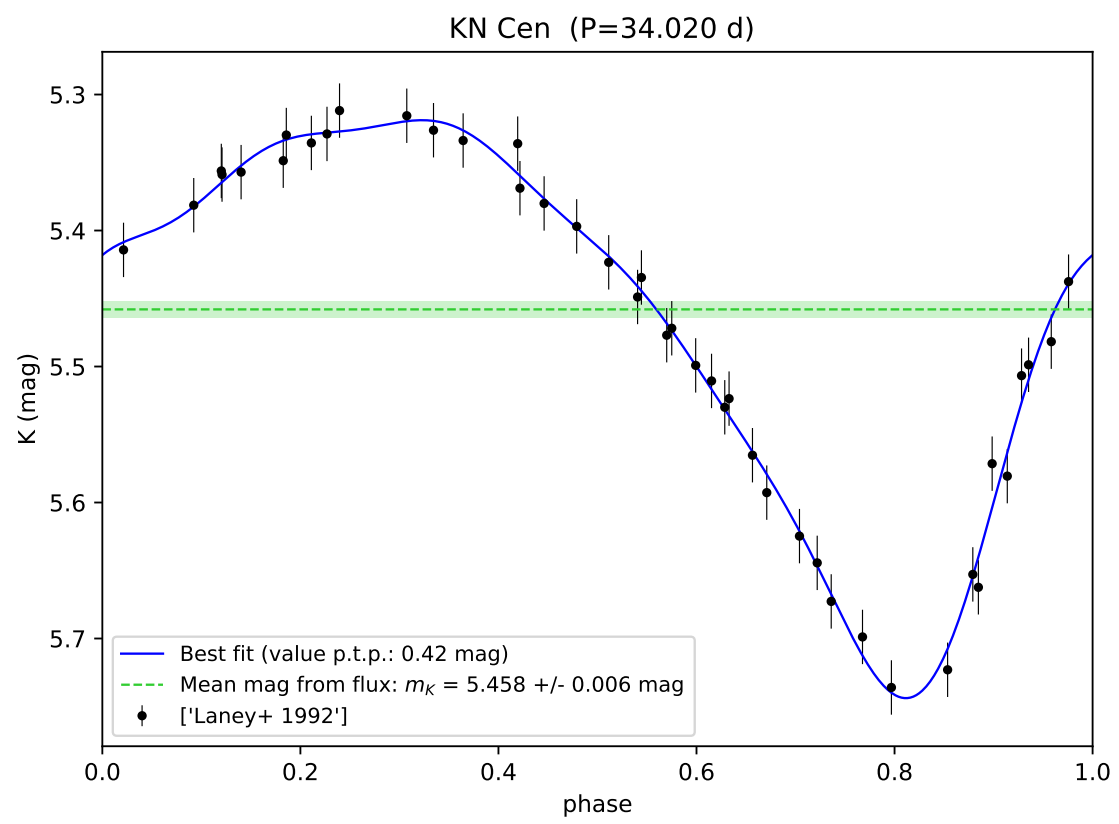

Figure 8. Example of a well covered light curve in the $K$ band for the Galactic Cepheid KN Cen. The solid blue line represents the best fit of the light curve, the dashed green line is the mean magnitude derived from the best fit and the green region is the uncertainty on the intensity-averaged mean magnitude.

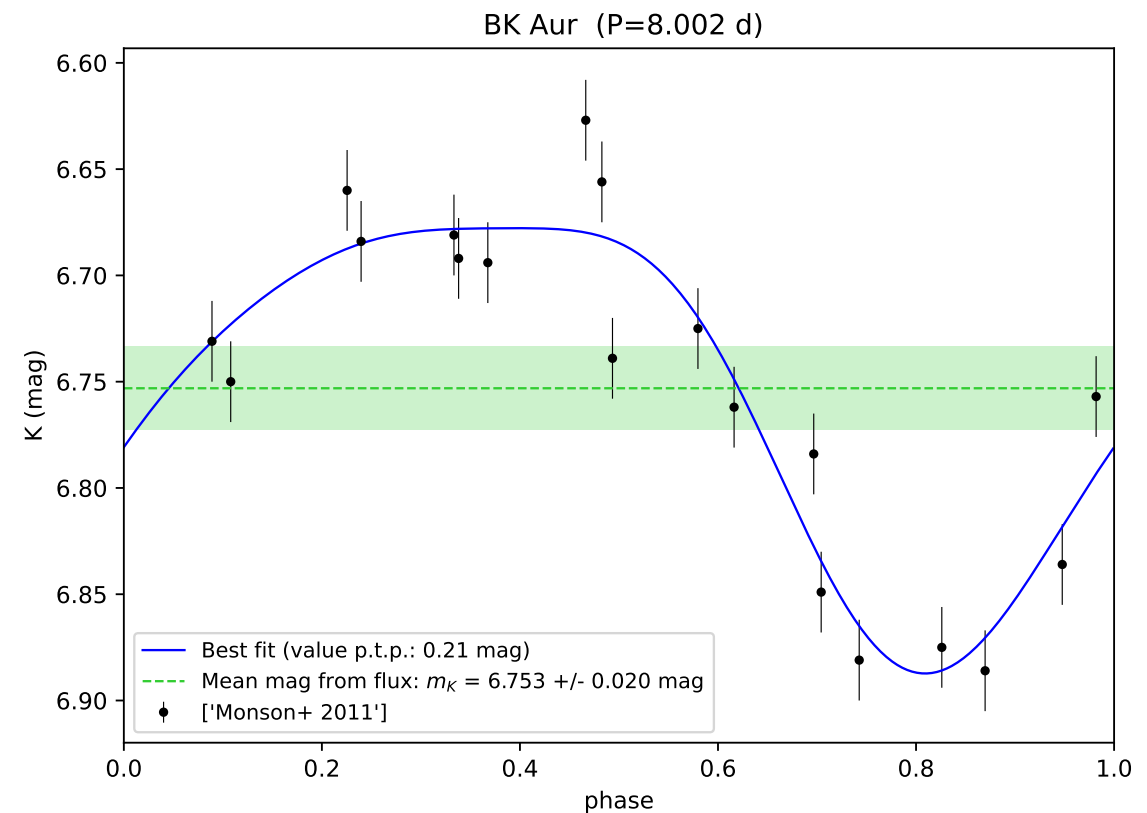

Figure 9. Example of a poor-quality light curve in the $K$ band for the Galactic Cepheid BK Aur. The solid blue line represents the best fit of the light curve, the dashed green line is the mean magnitude derived from the best fit and the green region is the uncertainty on the intensity-averaged mean magnitude. 
Table 3. Results of the PLZ linear fit of the form $M=\alpha(\log P-0.7)+\delta+\gamma[\mathrm{Fe} / \mathrm{H}]$ for Milky Way, Large Magellanic Cloud and Small Magellanic Cloud Cepheids fitted together for different conditions. The slope values are the same as in Table 2.

\begin{tabular}{lcccl}
\hline \hline Band & $\delta$ & $\gamma$ & $N$ & Comments \\
\hline$V$ & $-3.252 \pm 0.020$ & $-0.048 \pm 0.051$ & 1908 & Initial conditions $\left.{ }^{*}\right)$ \\
$I$ & $-3.948 \pm 0.020$ & $-0.138 \pm 0.051$ & 1907 & Initial conditions \\
$W_{V I}$ & $-5.005 \pm 0.022$ & $-0.251 \pm 0.057$ & 1864 & Initial conditions \\
$J$ & $-4.463 \pm 0.022$ & $-0.208 \pm 0.051$ & 1196 & Initial conditions \\
$H$ & $-4.748 \pm 0.020$ & $-0.152 \pm 0.092$ & 905 & Initial conditions \\
$K_{S}$ & $-4.826 \pm 0.019$ & $-0.221 \pm 0.050$ & 1199 & Initial conditions \\
$W_{J K}$ & $-5.075 \pm 0.022$ & $-0.214 \pm 0.057$ & 1198 & Initial conditions \\
\hline$V$ & $-3.274 \pm 0.020$ & $-0.084 \pm 0.051$ & 1908 & Gaia EDR3 parallax ZP $=-17 \mu$ as \\
$I$ & $-3.966 \pm 0.020$ & $-0.165 \pm 0.050$ & 1907 & Gaia EDR3 parallax ZP $=-17 \mu$ as \\
$W_{V I}$ & $-5.020 \pm 0.022$ & $-0.275 \pm 0.058$ & 1864 & Gaia EDR3 parallax ZP $=-17 \mu$ as \\
$J$ & $-4.495 \pm 0.022$ & $-0.258 \pm 0.052$ & 1196 & Gaia EDR3 parallax ZP $=-17 \mu$ as \\
$H$ & $-4.778 \pm 0.020$ & $-0.241 \pm 0.099$ & 905 & Gaia EDR3 parallax ZP $=-17 \mu$ as \\
$K_{S}$ & $-4.857 \pm 0.019$ & $-0.271 \pm 0.051$ & 1199 & Gaia EDR3 parallax ZP $=-17 \mu$ as \\
$W_{J K}$ & $-5.106 \pm 0.022$ & $-0.263 \pm 0.058$ & 1198 & Gaia EDR3 parallax ZP $=-17 \mu$ as \\
\hline$V$ & $-3.252 \pm 0.020$ & $-0.036 \pm 0.052$ & 1952 & $\mathrm{R}=0.7^{\circ}$ around SMC center \\
$I$ & $-3.948 \pm 0.020$ & $-0.130 \pm 0.052$ & 1951 & $\mathrm{R}=0.7^{\circ}$ around SMC center \\
$W_{V I}$ & $-5.005 \pm 0.022$ & $-0.252 \pm 0.058$ & 1908 & $\mathrm{R}=0.7^{\circ}$ around SMC center \\
$J$ & $-4.464 \pm 0.022$ & $-0.206 \pm 0.052$ & 1241 & $\mathrm{R}=0.7^{\circ}$ around SMC center \\
$K_{S}$ & $-4.826 \pm 0.019$ & $-0.218 \pm 0.051$ & 1244 & $\mathrm{R}=0.7^{\circ}$ around SMC center \\
$W_{J K}$ & $-5.075 \pm 0.022$ & $-0.208 \pm 0.058$ & 1242 & $\mathrm{R}=0.7^{\circ}$ around SMC center \\
\hline$V$ & $-3.250 \pm 0.020$ & $-0.077 \pm 0.051$ & 1845 & $\mathrm{R}=0.5^{\circ}$ around SMC center \\
$I$ & $-3.947 \pm 0.020$ & $-0.160 \pm 0.053$ & 1842 & $\mathrm{R}=0.5^{\circ}$ around SMC center \\
$W_{V I}$ & $-5.005 \pm 0.022$ & $-0.251 \pm 0.059$ & 1800 & $\mathrm{R}=0.5^{\circ}$ around SMC center \\
$J$ & $-4.462 \pm 0.022$ & $-0.225 \pm 0.054$ & 1132 & $\mathrm{R}=0.5^{\circ}$ around SMC center \\
$K_{S}$ & $-4.825 \pm 0.019$ & $-0.234 \pm 0.050$ & 1132 & $\mathrm{R}=0.5^{\circ}$ around SMC center \\
$W_{J K}$ & $-5.075 \pm 0.022$ & $-0.222 \pm 0.057$ & 1132 & $\mathrm{R}=0.5^{\circ}$ around SMC center \\
\hline & & & &
\end{tabular}

Note-(*) Initial conditions corresponds to Gaia EDR3 parallaxes corrected for individual zero-point and SMC Cepheids limited to a radius of 0.6 degree around the SMC center. 
Table 4. Sample of Milky Way Cepheids and main parameters. Parallaxes from Gaia EDR3 include zero point correction. Stars with a RUWE parameter larger than 1.4 were marked with a star and excluded from the sample. References: (F95): reddening from Fernie et al. (1995) multiplied by 0.94; (A12): reddening from Acharova et al. (2012); (G14): metallicity from Genovali et al. (2014); (G14b): metallicity from the literature (Genovali et al. 2013; Lemasle et al. 2007; Luck et al. 2011; Luck \& Lambert 2011; Pedicelli et al. 2010; Romaniello et al. 2008; Sziládi et al. 2007; Yong et al. 2006) rescaled to Genovali et al. (2014) solar abundance; (G15): metallicity from Genovali et al. (2015).

\begin{tabular}{|c|c|c|c|c|c|c|c|}
\hline Star & $\begin{array}{c}\text { Period } \\
\text { (day) }\end{array}$ & $\begin{array}{l}\pi_{\mathrm{EDR} 3} \\
(\mathrm{mas})\end{array}$ & RUWE & $\begin{array}{c}E(B-V) \\
(\mathrm{mag})\end{array}$ & Ref. & $\begin{array}{c}{[\mathrm{Fe} / \mathrm{H}]} \\
(\mathrm{dex})\end{array}$ & Ref. \\
\hline AA Gem & 11.302 & $0.311 \pm 0.018$ & 1.25 & $0.345 \pm 0.036$ & F95 & $-0.08 \pm 0.05$ & G15 \\
\hline AC Mon & 8.014 & $0.383 \pm 0.019$ & 1.38 & $0.507 \pm 0.033$ & F95 & $-0.03 \pm 0.06$ & G14b \\
\hline AD Gem & 3.788 & $0.370 \pm 0.020$ & 0.97 & $0.206 \pm 0.048$ & F95 & $-0.14 \pm 0.06$ & G15 \\
\hline AD Pup & 13.596 & $0.254 \pm 0.017$ & 1.36 & $0.363 \pm 0.020$ & F95 & $-0.20 \pm 0.15$ & G14b \\
\hline AE Vel & 7.134 & $0.369 \pm 0.012$ & 0.97 & $0.691 \pm 0.055$ & F95 & $0.14 \pm 0.06$ & G14b \\
\hline AG Cru & 3.837 & $0.758 \pm 0.020$ & 1.02 & $0.242 \pm 0.020$ & F95 & $0.08 \pm 0.06$ & G14b \\
\hline AP Pup & 5.084 & $0.924 \pm 0.020$ & 1.05 & $0.250 \pm 0.034$ & F95 & $-0.16 \pm 0.15$ & G14b \\
\hline AP Sgr & 5.058 & $1.217 \pm 0.024$ & 0.88 & $0.184 \pm 0.015$ & F95 & $0.10 \pm 0.08$ & G14b \\
\hline AQ Car & 9.769 & $0.361 \pm 0.016$ & 1.07 & $0.168 \pm 0.013$ & F95 & $-0.30 \pm 0.15$ & G14b \\
\hline AQ Pup & 30.149 & $0.294 \pm 0.023$ & 1.18 & $0.531 \pm 0.017$ & F95 & $0.06 \pm 0.05$ & G15 \\
\hline AS Per & 4.973 & $0.650 \pm 0.016$ & 1.08 & $0.684 \pm 0.041$ & F95 & $0.14 \pm 0.06$ & G14b \\
\hline AT Pup & 6.665 & $0.604 \pm 0.016$ & 1.04 & $0.166 \pm 0.011$ & F95 & $-0.22 \pm 0.15$ & G14b \\
\hline & & & & $1.238 \pm 0.027$ & F95 & $0.35 \pm 0.17$ & G15 \\
\hline & & & & & F95 & & G14b \\
\hline AY Cas & & & & & F95 & & G14b \\
\hline AY Cen & & & 0.95 & $0.357 \pm 0.066$ & F95 & $0.08 \pm 0.06$ & G14b \\
\hline AY Sgr & & & & $0.840 \pm 0.009$ & F95 & $0.11 \pm 0.06$ & G15 \\
\hline BB Her & & $0.280 \pm 0.015$ & & $0.392 \pm 0.039$ & $\mathrm{~A} 12$ & $0.26 \pm 0.06$ & G14b \\
\hline BB Sgr & & $1.188 \pm 0.024$ & 0.82 & $0.285 \pm 0.011$ & F95 & $0.08 \pm 0.08$ & G14b \\
\hline BE Mon & & $0.504 \pm 0.017$ & & $0.549 \pm 0.036$ & F95 & $0.05 \pm$ & G15 \\
\hline BF Oph & 4.068 & & 0.84 & $0.261 \pm 0.016$ & F95 & & G14b \\
\hline BG Vel & 6.924 & $1.045 \pm 0.017$ & 0.99 & $0.434 \pm 0.011$ & F95 & $-0.10 \pm 0.15$ & G14b \\
\hline BK Aur & & $0.426 \pm 0.015$ & 1.01 & $0.393 \pm 0.026$ & F95 & $-0.07 \pm 0.15$ & G14b \\
\hline BM Per & & $0.334 \pm 0.022$ & 0.99 & $0.919 \pm 0.059$ & F95 & $0.23 \pm 0.06$ & G14b \\
\hline BM Pup & & $0.302 \pm 0.013$ & 1.18 & $0.575 \pm 0.058$ & F95 & $-0.07 \pm 0.08$ & G15 \\
\hline BN Pup & 13.673 & $0.301 \pm 0.015$ & 1.25 & & F95 & $0.03 \pm 0.05$ & G15 \\
\hline BP Cas & & $0.442 \pm 0.013$ & 1.02 & & F95 & $0.09 \pm 0.06$ & $\mathrm{G} 14 \mathrm{~b}$ \\
\hline BZ Cyg & 10.142 & $0.500 \pm 0.014$ & & & F95 & $0.19 \pm 0.08$ & $\mathrm{G} 14 \mathrm{~b}$ \\
\hline CD Cas & 7.801 & $0.412 \pm 0.014$ & 1.06 & $0.745 \pm 0.012$ & F95 & $0.13 \pm 0.06$ & $\mathrm{G} 14 \mathrm{~b}$ \\
\hline CD Cyg & 17.074 & $0.394 \pm 0.016$ & 1.01 & $0.512 \pm 0.021$ & F95 & $0.15 \pm 0.06$ & G14b \\
\hline CE Pup & 49.326 & $0.114 \pm 0.014$ & 0.82 & $0.740 \pm 0.074$ & A12 & $-0.04 \pm 0.09$ & G14 \\
\hline CF Cas & 4.875 & $0.316 \pm 0.012$ & 1.04 & $0.556 \pm 0.021$ & F95 & $0.02 \pm 0.06$ & $\mathrm{G} 14 \mathrm{~b}$ \\
\hline CG Cas & 4.366 & $0.296 \pm 0.017$ & 1.03 & $0.667 \pm 0.009$ & F95 & $0.09 \pm 0.06$ & $\mathrm{G} 14 \mathrm{~b}$ \\
\hline CK Sct & 7.415 & $0.490 \pm 0.020$ & 0.97 & $0.816 \pm 0.024$ & F95 & $0.21 \pm 0.06$ & $\mathrm{G} 14 \mathrm{~b}$ \\
\hline CN Car & 4.933 & $0.342 \pm 0.014$ & 0.96 & $0.438 \pm 0.049$ & F95 & $0.21 \pm 0.06$ & $\mathrm{G} 14 \mathrm{~b}$ \\
\hline CP Cep & 17.859 & $0.279 \pm 0.021$ & 1.01 & $0.681 \pm 0.045$ & F95 & $-0.01 \pm 0.08$ & $\mathrm{G} 14 \mathrm{~b}$ \\
\hline CR Cep & 6.233 & $0.699 \pm 0.013$ & 1.06 & $0.704 \pm 0.009$ & F95 & $-0.06 \pm 0.08$ & $\mathrm{G} 14 \mathrm{~b}$ \\
\hline CR Ser & 5.301 & $0.578 \pm 0.020$ & 1.19 & $0.974 \pm 0.017$ & F95 & $0.12 \pm 0.08$ & G15 \\
\hline CS Mon & 6.732 & $0.324 \pm 0.014$ & 1.04 & $0.528 \pm 0.032$ & F95 & $-0.08 \pm 0.06$ & G14b \\
\hline CS Ori & 3.889 & $0.257 \pm 0.022$ & 1.33 & $0.373 \pm 0.030$ & F95 & $-0.25 \pm 0.06$ & G15 \\
\hline CS Vel & 5.905 & $0.272 \pm 0.016$ & 0.91 & $0.716 \pm 0.027$ & F95 & $0.12 \pm 0.06$ & G14b \\
\hline
\end{tabular}


Table 4 (continued)

\begin{tabular}{|c|c|c|c|c|c|c|c|}
\hline Star & $\begin{array}{c}\text { Period } \\
\text { (day) }\end{array}$ & $\begin{array}{c}\pi_{\text {EDR3 }} \\
\text { (mas) }\end{array}$ & RUWE & $\begin{array}{c}E(B-V) \\
\quad(\mathrm{mag})\end{array}$ & Ref. & $\begin{array}{c}{[\mathrm{Fe} / \mathrm{H}]} \\
(\mathrm{dex})\end{array}$ & Ref. \\
\hline CV Mon & 5.379 & $0.601 \pm 0.015$ & 1.10 & $0.705 \pm 0.018$ & F95 & $0.09 \pm 0.09$ & G15 \\
\hline CY Car & 4.266 & $0.427 \pm 0.011$ & 0.93 & $0.409 \pm 0.043$ & F95 & $0.11 \pm 0.06$ & G14b \\
\hline CY Cas & 14.377 & $0.255 \pm 0.019$ & 1.07 & $0.952 \pm 0.008$ & F95 & $0.06 \pm 0.08$ & G14b \\
\hline CZ Cas & 5.664 & $0.292 \pm 0.016$ & 0.96 & $0.761 \pm 0.030$ & F95 & $0.07 \pm 0.06$ & G14b \\
\hline DD Cas & 9.812 & $0.346 \pm 0.013$ & 1.05 & $0.486 \pm 0.016$ & F95 & $0.10 \pm 0.08$ & G14b \\
\hline DF Cas & 3.832 & $0.374 \pm 0.014$ & 1.05 & $0.564 \pm 0.049$ & F95 & $0.13 \pm 0.08$ & G14b \\
\hline DW Per & 3.650 & $0.296 \pm 0.019$ & 1.31 & $0.620 \pm 0.033$ & F95 & $-0.05 \pm 0.06$ & G14b \\
\hline EK Mon & 3.958 & $0.376 \pm 0.021$ & 1.16 & $0.547 \pm 0.003$ & F95 & $-0.05 \pm 0.15$ & G14b \\
\hline ER Car & 7.719 & $0.869 \pm 0.015$ & 0.82 & $0.111 \pm 0.016$ & F95 & $0.15 \pm 0.06$ & G14b \\
\hline EX Vel & 13.234 & $0.204 \pm 0.015$ & 0.94 & $0.728 \pm 0.052$ & F95 & $0.07 \pm 0.06$ & G14b \\
\hline FI Car & 13.458 & $0.242 \pm 0.019$ & 0.99 & $0.694 \pm 0.007$ & F95 & $0.31 \pm 0.06$ & G14b \\
\hline FM Aql & 6.114 & $1.014 \pm 0.026$ & 1.26 & $0.635 \pm 0.019$ & F95 & $0.24 \pm 0.06$ & G14b \\
\hline FN Aql & 9.482 & $0.736 \pm 0.025$ & 1.12 & $0.486 \pm 0.008$ & F95 & $-0.06 \pm 0.06$ & G14b \\
\hline GH Cyg & 7.818 & $0.417 \pm 0.014$ & 1.07 & $0.608 \pm 0.023$ & F95 & $0.21 \pm 0.06$ & G14b \\
\hline GI Cyg & 5.783 & $0.273 \pm 0.017$ & 1.01 & $0.734 \pm 0.073$ & F95 & $0.27 \pm 0.06$ & G14b \\
\hline GQ Ori & 8.616 & $0.408 \pm 0.021$ & 0.87 & $0.224 \pm 0.013$ & F95 & $0.20 \pm 0.08$ & G15 \\
\hline GU Nor & 3.453 & $0.565 \pm 0.015$ & 0.87 & $0.683 \pm 0.029$ & F95 & $0.08 \pm 0.06$ & G15 \\
\hline GX Car & 7.197 & $0.459 \pm 0.013$ & 1.02 & $0.380 \pm 0.008$ & F95 & $0.14 \pm 0.06$ & G14b \\
\hline GY Sge & 51.790 & $0.342 \pm 0.023$ & 0.95 & $1.183 \pm 0.111$ & F95 & $0.29 \pm 0.06$ & G14b \\
\hline HW Car & 9.199 & $0.397 \pm 0.012$ & 0.94 & $0.181 \pm 0.018$ & F95 & $0.09 \pm 0.06$ & G14b \\
\hline IQ Nor & 8.220 & $0.535 \pm 0.018$ & 0.97 & $0.676 \pm 0.044$ & F95 & $0.22 \pm 0.07$ & G15 \\
\hline IT Car & 7.533 & $0.702 \pm 0.020$ & 1.08 & $0.212 \pm 0.016$ & F95 & $0.14 \pm 0.06$ & G14b \\
\hline KK Cen & 12.180 & $0.152 \pm 0.018$ & 1.03 & $0.555 \pm 0.033$ & F95 & $0.24 \pm 0.06$ & G14b \\
\hline KN Cen & 34.020 & $0.251 \pm 0.018$ & 1.03 & $0.728 \pm 0.040$ & F95 & $0.55 \pm 0.12$ & G15 \\
\hline KQ Sco & 28.705 & $0.472 \pm 0.021$ & 0.91 & $0.852 \pm 0.041$ & F95 & $0.52 \pm 0.08$ & G15 \\
\hline LS Pup & 14.147 & $0.214 \pm 0.016$ & 1.25 & $0.452 \pm 0.009$ & F95 & $-0.12 \pm 0.11$ & G15 \\
\hline MW Cyg & 5.955 & $0.542 \pm 0.019$ & 1.21 & $0.651 \pm 0.039$ & F95 & $0.09 \pm 0.08$ & G14b \\
\hline MZ Cen & 10.354 & $0.221 \pm 0.017$ & 0.84 & $0.782 \pm 0.077$ & F95 & $0.27 \pm 0.10$ & G15 \\
\hline QY Cen & 17.752 & $0.293 \pm 0.021$ & 1.02 & $1.213 \pm 0.216$ & F95 & $0.24 \pm 0.06$ & G14b \\
\hline R Cru & 5.826 & $1.078 \pm 0.028$ & 1.16 & $0.156 \pm 0.012$ & F95 & $0.13 \pm 0.06$ & G14b \\
\hline R Mus & 7.510 & $1.076 \pm 0.018$ & 1.07 & $0.149 \pm 0.030$ & F95 & $-0.08 \pm 0.06$ & G14b \\
\hline $\mathrm{R} \operatorname{Tr} \mathrm{A}$ & 3.389 & $1.560 \pm 0.016$ & 0.89 & $0.167 \pm 0.025$ & F95 & $0.19 \pm 0.06$ & G14b \\
\hline RR Lac & 6.416 & $0.424 \pm 0.015$ & 1.10 & $0.267 \pm 0.023$ & F95 & $0.04 \pm 0.06$ & G14b \\
\hline RS Nor & 6.198 & $0.472 \pm 0.017$ & 0.94 & $0.577 \pm 0.036$ & F95 & $0.18 \pm 0.08$ & G15 \\
\hline RS Ori & 7.567 & $0.589 \pm 0.030$ & 1.12 & $0.332 \pm 0.010$ & F95 & $0.11 \pm 0.09$ & G15 \\
\hline RS Pup & 41.480 & $0.581 \pm 0.017$ & 1.16 & $0.451 \pm 0.010$ & F95 & $0.07 \pm 0.15$ & G14b \\
\hline RU Sct & 19.704 & $0.526 \pm 0.024$ & 0.87 & $0.914 \pm 0.017$ & F95 & $0.14 \pm 0.04$ & G15 \\
\hline RV Sco & 6.061 & $1.257 \pm 0.021$ & 0.81 & $0.343 \pm 0.007$ & F95 & $0.11 \pm 0.06$ & G14b \\
\hline RW Cas & 14.795 & $0.335 \pm 0.019$ & 1.26 & $0.440 \pm 0.032$ & F95 & $0.22 \pm 0.08$ & G14b \\
\hline RX Aur & 11.624 & $0.654 \pm 0.021$ & 0.98 & $0.254 \pm 0.020$ & F95 & $0.10 \pm 0.06$ & G14b \\
\hline RY CMa & 4.678 & $0.825 \pm 0.029$ & 1.29 & $0.238 \pm 0.016$ & F95 & $0.00 \pm 0.15$ & G14b \\
\hline RY Sco & 20.323 & $0.764 \pm 0.032$ & 0.73 & $0.654 \pm 0.044$ & F95 & $0.01 \pm 0.06$ & G15 \\
\hline RY Vel & 28.136 & $0.376 \pm 0.021$ & 1.07 & $0.539 \pm 0.012$ & F95 & $-0.05 \pm 0.15$ & G14b \\
\hline RZ Vel & 20.398 & $0.661 \pm 0.017$ & 1.24 & $0.301 \pm 0.011$ & F95 & $0.05 \pm 0.15$ & G14b \\
\hline S Cru & 4.690 & $1.342 \pm 0.024$ & 0.94 & $0.172 \pm 0.014$ & F95 & $0.11 \pm 0.06$ & G14b \\
\hline S Nor & 9.754 & $1.099 \pm 0.022$ & 0.88 & $0.182 \pm 0.008$ & F95 & $0.02 \pm 0.09$ & G14b \\
\hline $\mathrm{S} \operatorname{Tr} \mathrm{A}$ & 6.324 & $1.120 \pm 0.022$ & 1.04 & $0.086 \pm 0.010$ & F95 & $0.21 \pm 0.06$ & G14b \\
\hline
\end{tabular}


Breuval et AL.

Table 4 (continued)

\begin{tabular}{|c|c|c|c|c|c|c|c|}
\hline Star & $\begin{array}{l}\text { Period } \\
\text { (day) }\end{array}$ & $\begin{array}{l}\pi_{\mathrm{EDR} 3} \\
(\mathrm{mas})\end{array}$ & RUV & $\begin{array}{c}E(B-V) \\
(\mathrm{mag})\end{array}$ & Ref. & $\begin{array}{c}{[\mathrm{Fe} / \mathrm{H}]} \\
(\mathrm{dex})\end{array}$ & Ref. \\
\hline SS CMa & 12.361 & $307 \pm 0.013$ & 1.11 & $0.551 \pm 0.012$ & F95 & $0.06 \pm 0.04$ & G15 \\
\hline SS Sct & 671 & $0.934 \pm$ & & $340 \pm$ & F95 & 0.14 & G14b \\
\hline$\Gamma$ Tau & 034 & $0.916 \pm$ & 1.35 & $328 \pm 0.006$ & F95 & $-0.14 \pm$ & G14b \\
\hline Vel & 858 & $384 \pm$ & & $30 \pm$ & F95 & $-0.14 \pm$ & G14b \\
\hline SV Mon & .233 & $164 \pm 0.032$ & & $264 \pm 0.021$ & F95 & $0.12 \pm 0.08$ & G15 \\
\hline SV Vel & 14.097 & $0.434 \pm 0.018$ & 1.02 & $376 \pm 0.024$ & F95 & $0.12 \pm 0.06$ & G14b \\
\hline SV & 3 & 0.40 & & $74=$ & F95 & 0.05 & G14b \\
\hline SW Cas & 441 & $0.461 \pm 0.012$ & 12 & $475 \pm$ & F95 & $-0.03 \pm 0.08$ & G14b \\
\hline & 22407 & $2+0$ & & $338 \pm 0$ & F95 & $-0.15 \pm$ & G14b \\
\hline SX Car & .860 & $0.515 \pm 0$ & & $323 \pm 0$ & F95 & $0.05 \pm c$ & G14b \\
\hline SX & & & & $37=$ & F95 & -0 & G14b \\
\hline & 550 & & & $37 \pm$ & F95 & -0.18 & G14b \\
\hline & & & & $6 \pm$ & F95 & & $14 \mathrm{~b}$ \\
\hline SZ Aql & & 0 & & $53 \pm$ & F95 & 0.1 & G14b \\
\hline SZ Cas & & & & $13 \pm$ & F95 & 0.07 & G14b \\
\hline SZ Cyg & 10 & & & $4 \pm$ & F95 & 0.09 & G14b \\
\hline $\mathrm{T}$ Ant & & & & $00 \pm$ & $\mathrm{A} 12$ & $-0.20=$ & G14b \\
\hline T Cru & & & & $91 \pm$ & F95 & & G14b \\
\hline T Vel & 4.640 & & & $82 \pm$ & F95 & -0.02 & G14b \\
\hline T Vul & & & & 7 & F95 & $0.01 \pm$ & G14b \\
\hline & 1 & & & $37 \pm$ & F95 & $0.22 \pm$ & G14b \\
\hline & & & & $4 \pm$ & F95 & $0.01=$ & G15 \\
\hline TV & & & & $0 \pm$ & F95 & & G14b \\
\hline TW & 6. & & & $4 \pm$ & F95 & 0. & G15 \\
\hline & & & & $0 \pm$ & $\mathrm{F}^{\mathrm{S}}$ & 0.2 & G15 \\
\hline & & & & 8 & 5 & 2 & G15 \\
\hline & & & & 5 & F95 & & G14b \\
\hline TY & & & & $0 \pm$ & $\mathrm{F}$ & & G14b \\
\hline TZ Mon & & & & $34 \pm$ & F95 & -0.02 & G15 \\
\hline & & & & 0 & F95 & 0. & G14b \\
\hline U Car & 9 & 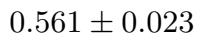 & & 3 & F95 & 99 & G14b \\
\hline U Nor & 12.644 & & & $868 \pm 0$. & F95 & $0.07 \pm 0.09$ & G14b \\
\hline & & & & $8 \pm$ & & 0.08 & G14b \\
\hline & 1 & 1 & & $1 \pm$ & F95 & 0.1 & G14b \\
\hline & & 0 & & 3 & F95 & 15 & G14b \\
\hline UX Per & & $0.162 \pm 0.020$ & & $62 \pm 0$ & F95 & $-0.05 \pm 0.06$ & G14b \\
\hline & & & & 7 & & & G14b \\
\hline & & & & 3 & Fs & 0. & G14b \\
\hline UZ Car & & & & 4 & F95 & 0. & $14 \mathrm{~b}$ \\
\hline UZ Cas & 4.259 & $0.251 \pm 0.020$ & & $469 \pm 0.034$ & F95 & $-0.05 \pm 0.06$ & G14b \\
\hline & 14.744 & .025 & & $59 \pm 0.023$ & F95 & $0.33 \pm 0.08$ & G15 \\
\hline $\mathrm{V} C \mathrm{C}_{\mathrm{a}}$ & 97 & $0.797 \pm 0.014$ & & $164 \pm 0.013$ & F95 & $-0.06 \pm 0.15$ & G14b \\
\hline V Cen & 5.494 & $1.409 \pm 0.022$ & & $265 \pm 0.016$ & F95 & $0.04 \pm 0.09$ & G14b \\
\hline V Lac & 4.983 & $0.496 \pm 0.016$ & 1.09 & $0.293 \pm 0.034$ & F95 & $0.06 \pm 0.06$ & G14b \\
\hline & & $3 \pm 0.017$ & & $0.225 \pm 0.021$ & F95 & $-0.30 \pm 0.15$ & G14b \\
\hline & & & & & Fs & & G15 \\
\hline V0340 Ara & 20.814 & $0.239 \pm 0.020$ & . & $0.548 \pm 0.008$ & F95 & $0.33 \pm 0.09$ & G15 \\
\hline
\end{tabular}


Table 4 (continued)

\begin{tabular}{|c|c|c|c|c|c|c|c|}
\hline Star & $\begin{array}{c}\text { Period } \\
\text { (day) }\end{array}$ & $\begin{array}{c}\pi_{\text {EDR3 }} \\
\text { (mas) }\end{array}$ & RUWE & $\begin{array}{c}E(B-V) \\
\quad(\mathrm{mag})\end{array}$ & Ref. & $\begin{array}{c}{[\mathrm{Fe} / \mathrm{H}]} \\
(\mathrm{dex})\end{array}$ & Ref. \\
\hline V0340 Nor & 11.289 & $0.491 \pm 0.025$ & 0.92 & $0.312 \pm 0.050$ & F95 & $0.07 \pm 0.07$ & G15 \\
\hline V0378 Cen & 6.460 & $0.524 \pm 0.019$ & 0.99 & $0.374 \pm 0.049$ & F95 & $0.08 \pm 0.06$ & G14b \\
\hline V0381 Cen & 5.079 & $0.818 \pm 0.020$ & 1.06 & $0.206 \pm 0.013$ & F95 & $0.02 \pm 0.06$ & G14b \\
\hline V0386 Cyg & 5.258 & $0.894 \pm 0.013$ & 0.95 & $0.907 \pm 0.033$ & F95 & $0.11 \pm 0.08$ & G14b \\
\hline V0402 Cyg & 4.365 & $0.410 \pm 0.011$ & 0.92 & $0.455 \pm 0.062$ & F95 & $0.02 \pm 0.08$ & G14b \\
\hline V0459 Cyg & 7.251 & $0.382 \pm 0.014$ & 1.09 & $0.775 \pm 0.024$ & F95 & $0.09 \pm 0.06$ & G14b \\
\hline V0470 Sco & 16.261 & $0.534 \pm c$ & 0.97 & $1.550 \pm 0.124$ & F95 & $0.16 \pm 0.06$ & G15 \\
\hline V0493 Aql & 2.988 & $0.472 \pm 0$ & 1.12 & $0.730 \pm 0.087$ & F95 & $0.03 \pm 0.06$ & G14b \\
\hline V0496 Cen & 4.424 & $0.563 \pm 0.013$ & 0.94 & $0.579 \pm 0.031$ & F95 & $0.09 \pm 0.06$ & G14b \\
\hline V0520 Cyg & 4.049 & $0.437 \pm 0$ & 1.03 & $0.754 \pm 0.075$ & F95 & $0.08 \pm 0.06$ & G14b \\
\hline V0538 Cyg & 6.119 & $0.394 \pm 0.017$ & 0.99 & $0.656 \pm 0.021$ & F95 & $0.05 \pm 0.06$ & G14b \\
\hline V0600 Aql & 7.239 & $0.523 \pm 0.019$ & 1.13 & $0.812 \pm 0.007$ & F95 & $0.03 \pm 0.08$ & G14b \\
\hline V0609 Cyg & 31.088 & $0.295 \pm 0.017$ & 1.12 & $1.243 \pm 0.124$ & F95 & $0.22 \pm 0.06$ & G14b \\
\hline V0636 Cas & 8.377 & $1.372 \pm 0.018$ & 1.02 & $0.593 \pm 0.065$ & F95 & $0.07 \pm 0.08$ & G14b \\
\hline V0636 Sco & 6.797 & $1.180 \pm 0.034$ & 1.15 & $0.227 \pm 0.017$ & F95 & $0.10 \pm 0.06$ & G14b \\
\hline V0733 Aql & 6.179 & $0.244 \pm 0.015$ & 0.98 & $0.106 \pm 0.011$ & $\mathrm{~A} 12$ & $0.08 \pm 0.08$ & G14b \\
\hline V0737 Cen & 7.066 & $1.213 \pm 0.019$ & 0.92 & $0.227 \pm 0.022$ & F95 & $0.14 \pm 0.06$ & G14b \\
\hline V1154 Cyg & 4.925 & $0.442 \pm 0.012$ & 1.04 & $0.315 \pm 0.031$ & F95 & $-0.10 \pm 0.08$ & G14b \\
\hline V1162 Aql & 5.376 & $0.823 \pm 0.023$ & 0.95 & $0.184 \pm 0.011$ & F95 & $0.01 \pm 0.08$ & G14b \\
\hline VW Cen & 15.036 & $0.260 \pm 0.016$ & 1.06 & $0.424 \pm 0.022$ & F95 & $0.41 \pm 0.08$ & G15 \\
\hline VW Cru & 5.265 & $0.738 \pm 0.016$ & 0.85 & $0.640 \pm 0.046$ & F95 & $0.19 \pm 0.06$ & G14b \\
\hline VY Car & 18.890 & $0.565 \pm 0.017$ & 0.92 & $0.270 \pm 0.019$ & F95 & $-0.06 \pm 0.15$ & G14b \\
\hline VY Cyg & 7.857 & $0.485 \pm 0.012$ & 1.07 & $0.596 \pm 0.021$ & F95 & $0.00 \pm 0.08$ & G14b \\
\hline VY Per & 5.532 & $0.485 \pm 0.017$ & 1.15 & $0.948 \pm 0.018$ & F95 & $0.04 \pm 0.06$ & G14b \\
\hline VY Sgr & 13.557 & $0.412 \pm 0.025$ & 0.81 & $0.903 \pm 0.243$ & F95 & $0.33 \pm 0.12$ & G15 \\
\hline VZ Cyg & 4.864 & $0.545 \pm 0.016$ & 1.31 & $0.291 \pm 0.015$ & F95 & $0.05 \pm 0.08$ & G14b \\
\hline VZ Pup & 23.175 & $0.220 \pm 0.015$ & 1.24 & $0.433 \pm 0.018$ & F95 & $-0.01 \pm 0.04$ & G15 \\
\hline W Gem & 7.914 & $1.006 \pm 0.028$ & 1.23 & $0.264 \pm 0.011$ & F95 & $0.02 \pm 0.06$ & G14b \\
\hline WW Pup & 5.517 & $0.212 \pm 0.016$ & 1.14 & $0.334 \pm 0.017$ & F95 & $0.13 \pm 0.16$ & G15 \\
\hline WX Pup & 8.937 & $0.387 \pm$ & 1.06 & $0.306 \pm 0.018$ & F95 & $-0.15 \pm 0.15$ & G14b \\
\hline WY Pup & 5.251 & $0.258 \pm 0.013$ & 1.02 & $0.259 \pm 0.031$ & F95 & $-0.10 \pm 0.08$ & G15 \\
\hline WZ Pup & 5.027 & $0.281 \pm 0.017$ & 1.35 & $0.196 \pm 0.022$ & F95 & $-0.07 \pm 0.06$ & G15 \\
\hline WZ Sgr & 21.851 & $0.612 \pm 0.028$ & 0.94 & $0.457 \pm 0.025$ & F95 & $0.28 \pm 0.08$ & G15 \\
\hline X Cru & 6.220 & $0.654 \pm 0.019$ & 0.95 & $0.294 \pm 0.019$ & F95 & $0.15 \pm 0.06$ & G14b \\
\hline X Cyg & 16.386 & $0.910 \pm 0.020$ & 1.28 & $0.251 \pm 0.010$ & F95 & $0.10 \pm 0.08$ & G14b \\
\hline X Pup & 25.973 & $0.397 \pm 0.020$ & 1.04 & $0.396 \pm 0.015$ & F95 & $0.02 \pm 0.08$ & G15 \\
\hline X Sct & 4.198 & $0.634 \pm 0.019$ & 0.80 & $0.581 \pm 0.030$ & F95 & $0.12 \pm 0.09$ & G15 \\
\hline X Sgr & 7.013 & $2.843 \pm 0.141$ & 1.22 & $0.189 \pm 0.020$ & F95 & $-0.21 \pm 0.30$ & G14b \\
\hline X Vul & 6.320 & $0.864 \pm 0.022$ & 1.06 & $0.775 \pm 0.021$ & F95 & $0.07 \pm 0.08$ & G14b \\
\hline XX Cen & 10.953 & $0.570 \pm 0.026$ & 1.24 & $0.245 \pm 0.012$ & F95 & $0.04 \pm 0.09$ & G14b \\
\hline XX Mon & 5.456 & $0.242 \pm 0.013$ & 0.86 & $0.586 \pm 0.014$ & F95 & $0.01 \pm 0.08$ & G15 \\
\hline XX Sgr & 6.424 & $0.724 \pm 0.027$ & 1.10 & $0.493 \pm 0.016$ & F95 & $-0.01 \pm 0.06$ & G15 \\
\hline XX Vel & 6.985 & $0.308 \pm 0.013$ & 0.88 & $0.530 \pm 0.007$ & F95 & $0.11 \pm 0.06$ & G14b \\
\hline XZ Car & 16.651 & $0.473 \pm 0.018$ & 1.05 & $0.372 \pm 0.026$ & F95 & $0.19 \pm 0.06$ & G14b \\
\hline Y Aur & 3.859 & $0.541 \pm 0.017$ & 1.12 & $0.384 \pm 0.031$ & F95 & $-0.26 \pm 0.15$ & G14b \\
\hline Y Lac & 4.324 & $0.431 \pm 0.013$ & 1.05 & $0.212 \pm 0.020$ & F95 & $0.03 \pm 0.06$ & G14b \\
\hline Y Oph & 17.125 & $1.348 \pm 0.036$ & 1.03 & $0.606 \pm 0.030$ & F95 & $0.06 \pm 0.08$ & G14b \\
\hline
\end{tabular}


Breuval et AL.

Table 4 (continued)

\begin{tabular}{lccccccc}
\hline Star & $\begin{array}{c}\text { Period } \\
\text { (day) }\end{array}$ & $\begin{array}{c}\pi_{\text {EDR3 }} \\
(\text { mas })\end{array}$ & RUWE & $\begin{array}{c}E(B-V) \\
(\mathrm{mag})\end{array}$ & Ref. & $\begin{array}{c}{[\mathrm{Fe} / \mathrm{H}]} \\
(\mathrm{dex})\end{array}$ & Ref. \\
\hline Y Sct & 10.342 & $0.558 \pm 0.020$ & 0.94 & $0.792 \pm 0.021$ & F95 & $0.23 \pm 0.06$ & G14b \\
YZ Aur & 18.193 & $0.233 \pm 0.016$ & 0.99 & $0.548 \pm 0.055$ & F95 & $-0.33 \pm 0.15$ & G14b \\
YZ Car & 18.168 & $0.358 \pm 0.018$ & 1.17 & $0.324 \pm 0.039$ & F95 & $0.00 \pm 0.06$ & G14b \\
YZ Sgr & 9.554 & $0.860 \pm 0.024$ & 0.95 & $0.289 \pm 0.007$ & F95 & $0.06 \pm 0.08$ & G14b \\
Z Lac & 10.886 & $0.510 \pm 0.021$ & 1.05 & $0.352 \pm 0.015$ & F95 & $0.10 \pm 0.06$ & G14b \\
Z Sct & 12.901 & $0.357 \pm 0.018$ & 0.90 & $0.535 \pm 0.039$ & F95 & $0.12 \pm 0.09$ & G15 \\
\hline
\end{tabular}


Table 5. Optical and NIR mean apparent magnitudes for the sample of Milky Way Cepheids. The magnitudes do not include the reddening correction. The uncertainties are only the random errors and do not include photometric zero point errors. References: (B97): Barnes et al. (1997); (F08): Feast et al. (2008); (L92): Laney \& Stobie (1992); (M11): Monson \& Pierce (2011); (W84): Welch et al. (1984). All magnitudes in $V$ and $I$ are from Berdnikov (2008).

\begin{tabular}{|c|c|c|c|c|c|c|}
\hline Star & $\begin{array}{c}V \\
\text { (mag) }\end{array}$ & $\begin{array}{c}I \\
(\mathrm{mag})\end{array}$ & $\begin{array}{c}J \\
(\mathrm{mag})\end{array}$ & $\begin{array}{c}H \\
(\mathrm{mag})\end{array}$ & $\begin{array}{c}K_{S} \\
(\mathrm{mag})\end{array}$ & Ref. NIR \\
\hline AA Gem & $9.735 \pm 0.008$ & - & $7.647 \pm 0.011$ & $7.206 \pm 0.010$ & $7.069 \pm 0.020$ & M11 \\
\hline AC Mon & $10.100 \pm 0.016$ & $8.708 \pm 0.012$ & $7.590 \pm 0.013$ & $7.072 \pm 0.011$ & $6.867 \pm 0.012$ & M11 \\
\hline AD Gem & - & - & $8.453 \pm 0.006$ & $8.154 \pm 0.006$ & $8.043 \pm 0.007$ & B97, M11 \\
\hline AD Pup & $9.898 \pm 0.006$ & $8.716 \pm 0.006$ & - & - & - & - \\
\hline AE Vel & $10.257 \pm 0.009$ & $8.730 \pm 0.007$ & - & - & - & - \\
\hline AG Cru & $8.228 \pm 0.006$ & $7.346 \pm 0.006$ & - & - & - & - \\
\hline AP Pup & $7.385 \pm 0.006$ & $6.463 \pm 0.006$ & - & - & - & - \\
\hline AP Sgr & $6.967 \pm 0.006$ & $6.053 \pm 0.006$ & - & - & - & - \\
\hline AQ Car & $8.892 \pm 0.023$ & $7.895 \pm 0.020$ & - & - & - & - \\
\hline AQ Pup & $8.690 \pm 0.006$ & $7.153 \pm 0.006$ & $6.000 \pm 0.006$ & $5.484 \pm 0.008$ & $5.256 \pm 0.009$ & L92 \\
\hline AS Per & - & - & $6.941 \pm 0.014$ & $6.482 \pm 0.007$ & $6.279 \pm 0.017$ & M11 \\
\hline AT Pup & $7.985 \pm 0.006$ & $7.080 \pm 0.006$ & - & - & - & - \\
\hline AV Sgr & $11.331 \pm 0.021$ & $8.851 \pm 0.013$ & - & - & - & - \\
\hline AW Per & $7.473 \pm 0.140$ & - & $5.222 \pm 0.010$ & $4.836 \pm 0.009$ & $4.676 \pm 0.010$ & M11 \\
\hline AX Cir & $5.887 \pm 0.006$ & $4.987 \pm 0.006$ & - & - & - & - \\
\hline AY Cas & $11.543 \pm 0.024$ & - & - & - & - & - \\
\hline AY Cen & $8.818 \pm 0.006$ & $7.701 \pm 0.006$ & - & - & - & - \\
\hline AY Sgr & $10.559 \pm 0.012$ & $8.729 \pm 0.009$ & $7.140 \pm 0.008$ & $6.534 \pm 0.010$ & $6.282 \pm 0.016$ & M11 \\
\hline BB Her & $10.093 \pm 0.007$ & $8.941 \pm 0.010$ & - & - & - & - \\
\hline BB Sgr & $6.952 \pm 0.006$ & $5.848 \pm 0.006$ & $5.025 \pm 0.006$ & $4.643 \pm 0.007$ & $4.496 \pm 0.008$ & L92, W84 \\
\hline BE Mon & $10.574 \pm 0.008$ & $9.243 \pm 0.008$ & $8.265 \pm 0.014$ & $7.857 \pm 0.011$ & $7.701 \pm 0.024$ & M11 \\
\hline BF Oph & $7.342 \pm 0.006$ & $6.367 \pm 0.006$ & $5.626 \pm 0.008$ & $5.298 \pm 0.007$ & $5.147 \pm 0.009$ & L92, W84 \\
\hline BG Lac & $8.897 \pm 0.006$ & $7.811 \pm 0.014$ & $7.023 \pm 0.006$ & $6.655 \pm 0.006$ & $6.500 \pm 0.006$ & B97, M11 \\
\hline BG Vel & $7.653 \pm 0.006$ & $6.342 \pm 0.006$ & - & - & - & - \\
\hline BK Aur & $9.445 \pm 0.015$ & - & $7.300 \pm 0.015$ & $6.890 \pm 0.019$ & $6.735 \pm 0.021$ & M11 \\
\hline BM Per & $10.428 \pm 0.028$ & - & $6.680 \pm 0.015$ & $6.007 \pm 0.012$ & $5.724 \pm 0.018$ & M11 \\
\hline BM Pup & $10.846 \pm 0.006$ & $9.414 \pm 0.006$ & - & - & - & - \\
\hline BN Pup & $9.907 \pm 0.016$ & $8.585 \pm 0.020$ & $7.534 \pm 0.008$ & $7.079 \pm 0.009$ & $6.880 \pm 0.008$ & L92 \\
\hline BP Cas & $10.951 \pm 0.021$ & - & - & - & - & - \\
\hline BZ Cyg & $10.221 \pm 0.006$ & $8.327 \pm 0.018$ & $6.774 \pm 0.014$ & $6.153 \pm 0.010$ & $5.879 \pm 0.014$ & M11 \\
\hline$\beta$ Dor & $3.737 \pm 0.006$ & $2.939 \pm 0.006$ & $2.365 \pm 0.006$ & $2.038 \pm 0.006$ & $1.925 \pm 0.006$ & F08, L92 \\
\hline CD Cas & $10.782 \pm 0.009$ & - & $7.644 \pm 0.006$ & $7.093 \pm 0.012$ & $6.878 \pm 0.012$ & M11 \\
\hline CD Cyg & $8.963 \pm 0.009$ & $7.498 \pm 0.028$ & $6.363 \pm 0.015$ & $5.853 \pm 0.012$ & $5.668 \pm 0.011$ & $\mathrm{~W} 84, \mathrm{M} 11$ \\
\hline CE Pup & $11.832 \pm 0.010$ & $9.968 \pm 0.007$ & - & - & - & - \\
\hline CF Cas & $11.138 \pm 0.006$ & $9.756 \pm 0.012$ & $8.606 \pm 0.010$ & $8.136 \pm 0.012$ & $7.923 \pm 0.012$ & $\mathrm{~W} 84, \mathrm{M} 11$ \\
\hline CG Cas & $11.378 \pm 0.010$ & - & - & - & - & - \\
\hline CK Sct & - & - & $7.393 \pm 0.006$ & $6.822 \pm 0.010$ & $6.610 \pm 0.014$ & M11 \\
\hline CN Car & $10.684 \pm 0.008$ & $9.355 \pm 0.009$ & - & - & - & - \\
\hline CP Cep & $10.588 \pm 0.012$ & $8.766 \pm 0.024$ & $7.348 \pm 0.010$ & $6.726 \pm 0.012$ & $6.492 \pm 0.012$ & M11 \\
\hline CR Cep & $9.646 \pm 0.008$ & $7.979 \pm 0.020$ & $6.654 \pm 0.006$ & $6.101 \pm 0.007$ & $5.890 \pm 0.007$ & M11 \\
\hline CR Ser & $10.857 \pm 0.009$ & $8.899 \pm 0.026$ & $7.353 \pm 0.007$ & $6.763 \pm 0.007$ & $6.503 \pm 0.012$ & M11 \\
\hline CS Mon & $11.005 \pm 0.006$ & $9.651 \pm 0.006$ & - & - & - & - \\
\hline CS Ori & $11.399 \pm 0.037$ & $10.261 \pm 0.019$ & $9.341 \pm 0.011$ & $8.960 \pm 0.009$ & $8.810 \pm 0.017$ & M11 \\
\hline CS Vel & $11.702 \pm 0.007$ & $10.069 \pm 0.007$ & $8.735 \pm 0.010$ & $8.228 \pm 0.014$ & $7.973 \pm 0.011$ & L92, W84 \\
\hline
\end{tabular}


Table 5 (continued)

\begin{tabular}{|c|c|c|c|c|c|c|}
\hline Star & $\begin{array}{c}V \\
(\mathrm{mag})\end{array}$ & $\begin{array}{c}I \\
(\mathrm{mag})\end{array}$ & $\begin{array}{c}J \\
(\mathrm{mag})\end{array}$ & $\begin{array}{c}H \\
(\mathrm{mag})\end{array}$ & $\begin{array}{c}K_{S} \\
(\mathrm{mag})\end{array}$ & Ref. NIR \\
\hline CV Mon & $10.291 \pm 0.006$ & $8.645 \pm 0.006$ & $7.323 \pm 0.011$ & $6.790 \pm 0.007$ & $6.545 \pm 0.007$ & L92, W84, M11 \\
\hline CY Car & $9.755 \pm 0.007$ & $8.712 \pm 0.006$ & - & - & - & - \\
\hline CY Cas & $11.643 \pm 0.020$ & - & $7.876 \pm 0.028$ & $7.180 \pm 0.018$ & $6.915 \pm 0.023$ & M11 \\
\hline CZ Cas & $11.752 \pm 0.009$ & $10.059 \pm 0.021$ & - & - & - & - \\
\hline DD Cas & $9.888 \pm 0.007$ & $8.561 \pm 0.025$ & $7.537 \pm 0.008$ & $7.073 \pm 0.011$ & $6.909 \pm 0.014$ & M11 \\
\hline DF Cas & $10.879 \pm 0.006$ & - & - & - & - & - \\
\hline DL Cas & $8.971 \pm 0.006$ & - & $6.560 \pm 0.014$ & $6.106 \pm 0.011$ & $5.912 \pm 0.015$ & W84, M11 \\
\hline DW Per & $11.577 \pm 0.008$ & - & - & - & - & - \\
\hline$\delta$ Cep & $3.930 \pm 0.010$ & - & $2.676 \pm 0.006$ & $2.393 \pm 0.006$ & $2.291 \pm 0.006$ & F08, B97 \\
\hline EK Mon & $11.062 \pm 0.006$ & $9.617 \pm 0.006$ & - & - & - & - \\
\hline ER Car & $6.828 \pm 0.006$ & $5.961 \pm 0.006$ & - & - & - & - \\
\hline EX Vel & $11.573 \pm 0.007$ & $9.775 \pm 0.006$ & - & - & - & - \\
\hline EY Car & $10.359 \pm 0.010$ & $9.260 \pm 0.008$ & - & - & - & - \\
\hline Eta Aql & $3.878 \pm 0.006$ & $3.024 \pm 0.006$ & $2.386 \pm 0.006$ & $2.067 \pm 0.006$ & $1.951 \pm 0.006$ & B97, W84 \\
\hline FI Car & $11.626 \pm 0.010$ & $9.855 \pm 0.009$ & - & - & - & - \\
\hline FM Aql & $8.278 \pm 0.006$ & $6.780 \pm 0.010$ & $5.681 \pm 0.006$ & $5.217 \pm 0.006$ & $5.026 \pm 0.006$ & B97, W84, M11 \\
\hline FN Aql & $8.383 \pm 0.006$ & $6.992 \pm 0.006$ & $5.965 \pm 0.006$ & $5.495 \pm 0.006$ & $5.315 \pm 0.006$ & B97, W84, M11 \\
\hline FN Vel & $10.303 \pm 0.006$ & $8.830 \pm 0.007$ & - & - & - & - \\
\hline GH Cyg & $9.904 \pm 0.006$ & $8.432 \pm 0.011$ & $7.262 \pm 0.011$ & $6.804 \pm 0.006$ & $6.598 \pm 0.016$ & M11 \\
\hline GI Cyg & $11.745 \pm 0.012$ & - & - & - & - & - \\
\hline GQ Ori & $8.965 \pm 0.011$ & $7.885 \pm 0.007$ & - & - & - & - \\
\hline GU Nor & $10.354 \pm 0.006$ & $8.799 \pm 0.007$ & - & - & - & - \\
\hline GX Car & $9.344 \pm 0.009$ & $8.137 \pm 0.006$ & - & - & - & - \\
\hline GY Sge & $10.163 \pm 0.006$ & - & $5.604 \pm 0.008$ & $4.887 \pm 0.007$ & $4.546 \pm 0.006$ & L92, W84 \\
\hline HW Car & $9.136 \pm 0.006$ & $8.028 \pm 0.006$ & - & - & - & - \\
\hline IQ Nor & $9.665 \pm 0.019$ & $8.115 \pm 0.020$ & - & - & - & - \\
\hline IT Car & $8.102 \pm 0.006$ & $7.070 \pm 0.006$ & - & - & - & - \\
\hline KK Cen & $11.452 \pm 0.036$ & $9.934 \pm 0.026$ & - & - & - & - \\
\hline KN Cen & $9.865 \pm 0.006$ & $7.994 \pm 0.006$ & $6.399 \pm 0.007$ & $5.747 \pm 0.008$ & $5.440 \pm 0.006$ & L92 \\
\hline KQ Sco & $9.835 \pm 0.006$ & $7.659 \pm 0.006$ & $5.909 \pm 0.012$ & $5.215 \pm 0.010$ & $4.901 \pm 0.013$ & L92, W84 \\
\hline$\ell$ Car & $3.723 \pm 0.006$ & $2.554 \pm 0.006$ & $1.679 \pm 0.006$ & $1.218 \pm 0.006$ & $1.054 \pm 0.006$ & L92 \\
\hline LS Pup & $10.462 \pm 0.007$ & $9.073 \pm 0.008$ & $7.999 \pm 0.006$ & $7.521 \pm 0.007$ & $7.312 \pm 0.006$ & L92 \\
\hline MW Cyg & $9.483 \pm 0.006$ & - & $6.700 \pm 0.006$ & $6.209 \pm 0.009$ & $5.998 \pm 0.014$ & M11 \\
\hline MZ Cen & $11.553 \pm 0.007$ & $9.786 \pm 0.010$ & - & - & - & - \\
\hline QY Cen & $11.784 \pm 0.006$ & $9.350 \pm 0.007$ & - & - & - & - \\
\hline $\mathrm{R}$ Cru & $6.771 \pm 0.006$ & $5.901 \pm 0.006$ & - & - & - & - \\
\hline R Mus & $6.313 \pm 0.006$ & $5.497 \pm 0.006$ & - & - & - & - \\
\hline $\mathrm{R} \operatorname{Tr} \mathrm{A}$ & $6.656 \pm 0.006$ & $5.843 \pm 0.006$ & - & - & - & - \\
\hline RR Lac & $8.846 \pm 0.006$ & $7.814 \pm 0.015$ & $6.977 \pm 0.008$ & $6.628 \pm 0.010$ & $6.488 \pm 0.011$ & M11 \\
\hline RS Nor & $10.019 \pm 0.018$ & $8.541 \pm 0.013$ & - & - & - & - \\
\hline RS Ori & $8.410 \pm 0.011$ & $7.282 \pm 0.012$ & $6.408 \pm 0.016$ & $6.027 \pm 0.017$ & $5.880 \pm 0.019$ & M11 \\
\hline RS Pup & $7.008 \pm 0.006$ & $5.478 \pm 0.006$ & $4.341 \pm 0.009$ & $3.830 \pm 0.007$ & $3.605 \pm 0.008$ & L92, W84 \\
\hline RT Aur & $5.469 \pm 0.076$ & $4.811 \pm 0.043$ & $4.236 \pm 0.008$ & $3.998 \pm 0.007$ & $3.906 \pm 0.006$ & B97, M11 \\
\hline RU Sct & - & - & $5.909 \pm 0.008$ & $5.298 \pm 0.007$ & $5.036 \pm 0.009$ & L92, W84, M11 \\
\hline RV Sco & $7.046 \pm 0.006$ & $5.907 \pm 0.006$ & - & - & - & - \\
\hline RW Cam & $8.657 \pm 0.010$ & - & $5.828 \pm 0.012$ & $5.291 \pm 0.013$ & $5.093 \pm 0.010$ & M11 \\
\hline RW Cas & $9.248 \pm 0.019$ & $7.871 \pm 0.020$ & $6.841 \pm 0.024$ & $6.372 \pm 0.011$ & $6.194 \pm 0.026$ & M11 \\
\hline
\end{tabular}


Table 5 (continued)

\begin{tabular}{|c|c|c|c|c|c|c|}
\hline Star & $\begin{array}{c}V \\
\text { (mag) }\end{array}$ & $\begin{array}{c}I \\
(\mathrm{mag})\end{array}$ & $\begin{array}{c}J \\
(\mathrm{mag})\end{array}$ & $\begin{array}{c}H \\
(\mathrm{mag})\end{array}$ & $\begin{array}{c}K_{S} \\
(\mathrm{mag})\end{array}$ & Ref. NIR \\
\hline RX Aur & $7.670 \pm 0.007$ & - & $5.737 \pm 0.008$ & $5.363 \pm 0.011$ & $5.233 \pm 0.017$ & M11 \\
\hline RX Cam & $7.670 \pm 0.012$ & 一 & $5.178 \pm 0.023$ & $4.732 \pm 0.020$ & $4.561 \pm 0.012$ & M11 \\
\hline $\mathrm{RY} \mathrm{CMa}$ & $8.109 \pm 0.006$ & $7.133 \pm 0.006$ & 一 & - & - & - \\
\hline RY Sco & $7.999 \pm 0.006$ & $6.253 \pm 0.006$ & $4.899 \pm 0.006$ & $4.368 \pm 0.006$ & $4.102 \pm 0.007$ & L92, W84 \\
\hline RY Vel & $8.376 \pm 0.006$ & $6.827 \pm 0.006$ & $5.604 \pm 0.008$ & $5.124 \pm 0.007$ & $4.886 \pm 0.006$ & L92, W84 \\
\hline $\mathrm{RZ} \mathrm{CMa}$ & $9.702 \pm 0.007$ & $8.504 \pm 0.007$ & 一 & - & - & - \\
\hline RZ Gem & $10.048 \pm 0.249$ & - & $7.612 \pm 0.010$ & $7.169 \pm 0.009$ & $6.970 \pm 0.015$ & M11 \\
\hline RZ Vel & $7.089 \pm 0.006$ & $5.862 \pm 0.006$ & $4.889 \pm 0.012$ & $4.463 \pm 0.007$ & $4.267 \pm 0.006$ & L92 \\
\hline S Cru & $6.601 \pm 0.006$ & $5.732 \pm 0.006$ & 一 & - & - & - \\
\hline S Mus & $6.133 \pm 0.006$ & $5.199 \pm 0.006$ & $4.473 \pm 0.006$ & $4.135 \pm 0.006$ & $3.983 \pm 0.008$ & L92, W84 \\
\hline S Nor & $6.427 \pm 0.006$ & $5.428 \pm 0.006$ & $4.652 \pm 0.006$ & $4.286 \pm 0.006$ & $4.131 \pm 0.008$ & L92, W84 \\
\hline S Sge & $5.612 \pm 0.006$ & $4.772 \pm 0.010$ & $4.155 \pm 0.006$ & $3.847 \pm 0.006$ & $3.732 \pm 0.006$ & W84, B97 \\
\hline $\mathrm{S} \operatorname{Tr} \mathrm{A}$ & $6.391 \pm 0.006$ & $5.592 \pm 0.006$ & - & - & - & 一 \\
\hline SS CMa & - & $8.480 \pm 0.010$ & - & - & - & 一 \\
\hline SS Sct & - & - & $6.299 \pm 0.008$ & $5.938 \pm 0.006$ & $5.807 \pm 0.008$ & W84, M11 \\
\hline ST Tau & $8.243 \pm 0.014$ & $7.171 \pm 0.016$ & - & - & - & - \\
\hline ST Vel & $9.699 \pm 0.006$ & $8.286 \pm 0.006$ & - & - & - & - \\
\hline SU Cyg & $6.855 \pm 0.007$ & $6.198 \pm 0.013$ & $5.638 \pm 0.007$ & $5.397 \pm 0.007$ & $5.295 \pm 0.008$ & W84, M11 \\
\hline SV Mon & $8.266 \pm 0.008$ & $7.139 \pm 0.006$ & $6.262 \pm 0.015$ & $5.835 \pm 0.010$ & $5.691 \pm 0.017$ & M11 \\
\hline SV Per & $8.977 \pm 0.011$ & - & $6.802 \pm 0.021$ & $6.360 \pm 0.016$ & $6.198 \pm 0.018$ & M11 \\
\hline SV Vel & $8.583 \pm 0.006$ & $7.329 \pm 0.006$ & 一 & - & - & - \\
\hline SV Vul & $7.216 \pm 0.006$ & $5.697 \pm 0.009$ & $4.571 \pm 0.006$ & $4.077 \pm 0.006$ & $3.887 \pm 0.006$ & W84, L92, B97, M11 \\
\hline SW Cas & $9.713 \pm 0.007$ & $8.438 \pm 0.020$ & $7.412 \pm 0.009$ & $6.987 \pm 0.013$ & $6.820 \pm 0.015$ & M11 \\
\hline SW Vel & $8.137 \pm 0.014$ & $6.850 \pm 0.008$ & $5.852 \pm 0.018$ & $5.407 \pm 0.012$ & $5.203 \pm 0.011$ & L92 \\
\hline SX Car & $9.082 \pm 0.006$ & $8.039 \pm 0.006$ & 一 & - & - & - \\
\hline SX Per & $11.223 \pm 0.104$ & - & $8.769 \pm 0.010$ & $8.352 \pm 0.007$ & $8.187 \pm 0.013$ & M11 \\
\hline SX Vel & $8.278 \pm 0.006$ & $7.262 \pm 0.006$ & $6.474 \pm 0.006$ & $6.127 \pm 0.006$ & $5.965 \pm 0.006$ & L92 \\
\hline SY Aur & $9.069 \pm 0.009$ & - & $6.923 \pm 0.009$ & $6.530 \pm 0.012$ & $6.367 \pm 0.014$ & M11 \\
\hline SY Nor & $9.520 \pm 0.023$ & $7.949 \pm 0.030$ & 一 & - & - & - \\
\hline SZ Aql & $8.636 \pm 0.011$ & $7.082 \pm 0.015$ & $5.865 \pm 0.008$ & $5.351 \pm 0.006$ & $5.138 \pm 0.006$ & B97, W84, L92, M11 \\
\hline SZ Cas & $9.843 \pm 0.006$ & $8.110 \pm 0.008$ & 一 & - & - & - \\
\hline SZ Cyg & $9.435 \pm 0.011$ & $7.798 \pm 0.026$ & $6.530 \pm 0.009$ & $5.960 \pm 0.007$ & $5.732 \pm 0.014$ & M11 \\
\hline $\mathrm{T}$ Ant & $9.331 \pm 0.006$ & $8.523 \pm 0.006$ & 一 & - & - & 一 \\
\hline T Cru & $6.570 \pm 0.006$ & $5.608 \pm 0.006$ & 一 & - & - & - \\
\hline T Mon & $6.138 \pm 0.006$ & $4.987 \pm 0.006$ & $4.092 \pm 0.009$ & $3.648 \pm 0.009$ & $3.487 \pm 0.008$ & W84, L92, M11 \\
\hline $\mathrm{T}$ Vel & $8.029 \pm 0.006$ & $6.964 \pm 0.006$ & $6.143 \pm 0.006$ & $5.775 \pm 0.006$ & $5.605 \pm 0.006$ & L92 \\
\hline T Vul & $5.750 \pm 0.006$ & $5.077 \pm 0.015$ & $4.532 \pm 0.006$ & $4.272 \pm 0.006$ & $4.174 \pm 0.006$ & W84, B97 \\
\hline TT Aql & $7.141 \pm 0.006$ & $5.732 \pm 0.009$ & $4.671 \pm 0.009$ & $4.194 \pm 0.007$ & $4.017 \pm 0.006$ & W84, M11, B97 \\
\hline TV CMa & $10.587 \pm 0.011$ & $9.173 \pm 0.011$ & $8.035 \pm 0.008$ & $7.588 \pm 0.011$ & $7.386 \pm 0.014$ & M11 \\
\hline TV Cam & $11.729 \pm 0.018$ & - & 一 & - & - & - \\
\hline TW CMa & $9.573 \pm 0.007$ & $8.458 \pm 0.007$ & $7.577 \pm 0.010$ & $7.183 \pm 0.009$ & $7.029 \pm 0.017$ & M11 \\
\hline TW Nor & $11.670 \pm 0.007$ & $9.306 \pm 0.010$ & $7.406 \pm 0.022$ & $6.705 \pm 0.013$ & $6.358 \pm 0.029$ & L92, W84 \\
\hline TX Cen & $10.527 \pm 0.006$ & $8.618 \pm 0.006$ & 一 & - & - & - \\
\hline TX Cyg & $9.490 \pm 0.012$ & $7.225 \pm 0.030$ & $5.342 \pm 0.020$ & $4.633 \pm 0.018$ & $4.323 \pm 0.018$ & M11 \\
\hline TX Mon & $10.960 \pm 0.010$ & $9.634 \pm 0.008$ & $8.581 \pm 0.013$ & $8.121 \pm 0.013$ & $7.943 \pm 0.017$ & M11 \\
\hline TY Sct & $10.821 \pm 0.013$ & $8.811 \pm 0.016$ & $7.247 \pm 0.011$ & $6.637 \pm 0.009$ & $6.386 \pm 0.021$ & M11 \\
\hline TZ Mon & $10.793 \pm 0.008$ & $9.472 \pm 0.006$ & $8.458 \pm 0.012$ & $8.009 \pm 0.014$ & $7.815 \pm 0.016$ & M11 \\
\hline
\end{tabular}


Table 5 (continued)

\begin{tabular}{|c|c|c|c|c|c|c|}
\hline Star & $\begin{array}{c}V \\
(\mathrm{mag})\end{array}$ & $\begin{array}{c}I \\
(\mathrm{mag})\end{array}$ & $\begin{array}{c}J \\
(\mathrm{mag})\end{array}$ & $\begin{array}{c}H \\
(\mathrm{mag})\end{array}$ & $\begin{array}{c}K_{S} \\
(\mathrm{mag})\end{array}$ & Ref. NIR \\
\hline TZ Mus & $11.690 \pm 0.006$ & $10.144 \pm 0.008$ & - & - & - & - \\
\hline U Aql & $6.432 \pm 0.006$ & $5.271 \pm 0.010$ & $4.389 \pm 0.012$ & $3.999 \pm 0.009$ & $3.844 \pm 0.010$ & W84, M11 \\
\hline U Car & $6.284 \pm 0.006$ & $5.052 \pm 0.006$ & $4.104 \pm 0.007$ & $3.674 \pm 0.006$ & $3.483 \pm 0.006$ & L92, W84 \\
\hline U Nor & - & - & $5.825 \pm 0.006$ & $5.236 \pm 0.006$ & $4.944 \pm 0.006$ & L92 \\
\hline U Sgr & $6.697 \pm 0.006$ & $5.436 \pm 0.006$ & $4.512 \pm 0.006$ & $4.100 \pm 0.006$ & $3.933 \pm 0.007$ & W84, L92, M11 \\
\hline U Vul & $7.122 \pm 0.006$ & $5.602 \pm 0.011$ & $4.528 \pm 0.009$ & $4.093 \pm 0.007$ & $3.912 \pm 0.006$ & B97, M11 \\
\hline UU Mus & - & - & $7.439 \pm 0.007$ & $6.994 \pm 0.006$ & $6.788 \pm 0.006$ & L92 \\
\hline UW Car & $9.424 \pm 0.008$ & $8.218 \pm 0.013$ & - & - & - & - \\
\hline UX Car & $8.295 \pm 0.006$ & $7.554 \pm 0.006$ & - & - & - & - \\
\hline UX Per & $11.650 \pm 0.018$ & - & - & - & - & - \\
\hline UY Car & $8.948 \pm 0.006$ & $8.007 \pm 0.010$ & - & - & - & - \\
\hline UY Per & $11.319 \pm 0.013$ & $9.493 \pm 0.016$ & - & - & - & - \\
\hline UZ Car & $9.327 \pm 0.006$ & $8.365 \pm 0.006$ & - & - & - & - \\
\hline UZ Cas & $11.379 \pm 0.007$ & - & - & - & - & - \\
\hline UZ Sct & $11.289 \pm 0.022$ & $9.148 \pm 0.035$ & $7.418 \pm 0.010$ & $6.741 \pm 0.010$ & $6.485 \pm 0.016$ & M11 \\
\hline V Car & $7.368 \pm 0.006$ & $6.433 \pm 0.006$ & $5.728 \pm 0.006$ & $5.396 \pm 0.006$ & $5.249 \pm 0.006$ & L92 \\
\hline V Cen & $6.830 \pm 0.006$ & $5.794 \pm 0.006$ & $4.995 \pm 0.006$ & $4.638 \pm 0.006$ & $4.479 \pm 0.009$ & L92, W84 \\
\hline V Lac & $8.932 \pm 0.007$ & - & - & - & - & - \\
\hline V Vel & $7.586 \pm 0.006$ & $6.691 \pm 0.006$ & - & - & - & - \\
\hline V0339 Cen & $8.714 \pm 0.013$ & $7.384 \pm 0.010$ & - & - & - & - \\
\hline V0340 Ara & $10.228 \pm 0.014$ & $8.580 \pm 0.007$ & - & - & - & - \\
\hline V0340 Nor & $8.403 \pm 0.008$ & $7.167 \pm 0.008$ & - & - & - & - \\
\hline V0350 Sgr & $7.481 \pm 0.006$ & $6.435 \pm 0.006$ & $5.627 \pm 0.011$ & $5.256 \pm 0.011$ & $5.130 \pm 0.008$ & W84 \\
\hline V0378 Cen & $8.479 \pm 0.006$ & $7.260 \pm 0.006$ & - & - & - & - \\
\hline V0381 Cen & $7.675 \pm 0.006$ & $6.791 \pm 0.006$ & - & - & - & - \\
\hline V0386 Cyg & $9.624 \pm 0.007$ & - & $6.375 \pm 0.007$ & $5.809 \pm 0.007$ & $5.540 \pm 0.015$ & M11 \\
\hline V0395 Cas & $10.748 \pm 0.019$ & $9.447 \pm 0.035$ & - & - & - & - \\
\hline V0402 Cyg & $9.864 \pm 0.006$ & - & $7.809 \pm 0.006$ & $7.416 \pm 0.006$ & $7.263 \pm 0.015$ & M11 \\
\hline V0459 Cyg & $10.576 \pm 0.033$ & $8.881 \pm 0.019$ & $7.613 \pm 0.011$ & $7.075 \pm 0.010$ & $6.859 \pm 0.016$ & M11 \\
\hline V0470 Sco & $11.005 \pm 0.008$ & $8.246 \pm 0.007$ & - & - & - & - \\
\hline V0493 Aql & $11.046 \pm 0.006$ & - & - & - & - & - \\
\hline V0496 Aql & $7.769 \pm 0.006$ & $6.489 \pm 0.008$ & - & - & - & - \\
\hline V0496 Cen & $9.945 \pm 0.006$ & $8.539 \pm 0.006$ & - & - & - & - \\
\hline V0508 Mon & $10.502 \pm 0.006$ & $9.461 \pm 0.006$ & - & - & - & - \\
\hline V0520 Cyg & $10.852 \pm 0.006$ & $9.306 \pm 0.029$ & - & - & - & - \\
\hline V0538 Cyg & $10.449 \pm 0.009$ & $8.971 \pm 0.049$ & $7.803 \pm 0.007$ & $7.311 \pm 0.006$ & $7.119 \pm 0.008$ & M11 \\
\hline V0600 Aql & $10.034 \pm 0.006$ & $8.281 \pm 0.011$ & - & - & - & - \\
\hline V0609 Cyg & $11.026 \pm 0.017$ & $8.683 \pm 0.015$ & $6.832 \pm 0.013$ & $6.128 \pm 0.010$ & $5.800 \pm 0.017$ & M11 \\
\hline V0636 Cas & $7.183 \pm 0.006$ & - & - & - & - & - \\
\hline V0636 Sco & $6.654 \pm 0.006$ & $5.649 \pm 0.006$ & - & - & - & - \\
\hline V0733 Aql & $9.976 \pm 0.006$ & $9.040 \pm 0.012$ & - & - & - & - \\
\hline V0737 Cen & $6.724 \pm 0.006$ & $5.701 \pm 0.006$ & - & - & - & - \\
\hline V1154 Cyg & $9.186 \pm 0.006$ & $8.180 \pm 0.018$ & - & - & - & - \\
\hline V1162 Aql & $7.806 \pm 0.006$ & $6.850 \pm 0.007$ & $6.143 \pm 0.008$ & $5.814 \pm 0.017$ & $5.682 \pm 0.020$ & M11 \\
\hline VV Cas & $10.768 \pm 0.016$ & $9.432 \pm 0.018$ & $8.328 \pm 0.008$ & $7.885 \pm 0.006$ & $7.719 \pm 0.008$ & M11 \\
\hline VW Cen & $10.263 \pm 0.007$ & $8.783 \pm 0.006$ & $7.555 \pm 0.007$ & $7.015 \pm 0.006$ & $6.775 \pm 0.006$ & L92 \\
\hline VW Cru & $9.597 \pm 0.009$ & $7.977 \pm 0.006$ & - & - & - & - \\
\hline
\end{tabular}


Table 5 (continued)

\begin{tabular}{|c|c|c|c|c|c|c|}
\hline Star & $\begin{array}{c}V \\
\text { (mag) }\end{array}$ & $\begin{array}{c}I \\
(\mathrm{mag})\end{array}$ & $\begin{array}{c}J \\
(\mathrm{mag}) \\
\end{array}$ & $\begin{array}{c}H \\
(\mathrm{mag})\end{array}$ & $\begin{array}{c}K_{S} \\
(\mathrm{mag})\end{array}$ & Ref. NIR \\
\hline VW Pup & $11.393 \pm 0.007$ & $10.091 \pm 0.006$ & 一 & - & 一 & 一 \\
\hline VY Car & $7.460 \pm 0.006$ & $6.283 \pm 0.006$ & $5.375 \pm 0.015$ & $4.943 \pm 0.010$ & $4.760 \pm 0.010$ & L92, W84 \\
\hline VY Cyg & $9.594 \pm 0.006$ & $8.127 \pm 0.019$ & $7.009 \pm 0.006$ & $6.552 \pm 0.009$ & $6.355 \pm 0.010$ & M11 \\
\hline VY Per & $11.221 \pm 0.014$ & $9.297 \pm 0.026$ & - & - & - & 一 \\
\hline VY Sgr & $11.469 \pm 0.012$ & $9.129 \pm 0.013$ & - & - & - & 一 \\
\hline VZ Cyg & $8.970 \pm 0.008$ & $7.966 \pm 0.015$ & $7.201 \pm 0.007$ & $6.864 \pm 0.006$ & $6.721 \pm 0.006$ & B97, W84, M11 \\
\hline VZ Pup & $9.657 \pm 0.009$ & $8.302 \pm 0.006$ & $7.277 \pm 0.007$ & $6.830 \pm 0.006$ & $6.626 \pm 0.006$ & L92 \\
\hline W Gem & $7.012 \pm 0.049$ & - & $5.129 \pm 0.033$ & $4.771 \pm 0.026$ & $4.656 \pm 0.021$ & M11 \\
\hline W Sgr & $4.664 \pm 0.006$ & $3.850 \pm 0.006$ & - & - & - & 一 \\
\hline WW Car & $9.750 \pm 0.010$ & $8.644 \pm 0.007$ & - & - & - & 一 \\
\hline WW Pup & $10.599 \pm 0.010$ & $9.525 \pm 0.007$ & - & - & - & - \\
\hline WX Pup & $9.070 \pm 0.007$ & $7.968 \pm 0.006$ & - & - & - & - \\
\hline WY Pup & $10.599 \pm 0.013$ & $9.662 \pm 0.008$ & - & - & - & - \\
\hline WZ Pup & $10.320 \pm 0.006$ & $9.424 \pm 0.006$ & - & - & - & - \\
\hline WZ Sgr & $8.046 \pm 0.011$ & $6.544 \pm 0.010$ & $5.282 \pm 0.008$ & $4.761 \pm 0.006$ & $4.538 \pm 0.008$ & L92, W84, M11 \\
\hline $\mathrm{X}$ Cru & $8.404 \pm 0.006$ & - & - & - & - & - \\
\hline X Cyg & $6.385 \pm 0.009$ & $5.236 \pm 0.028$ & $4.383 \pm 0.008$ & $3.960 \pm 0.006$ & $3.799 \pm 0.006$ & W84, B97 \\
\hline X Pup & $8.517 \pm 0.011$ & $7.161 \pm 0.006$ & $6.077 \pm 0.023$ & $5.599 \pm 0.011$ & $5.386 \pm 0.011$ & L92 \\
\hline X Sct & $10.031 \pm 0.017$ & $8.613 \pm 0.033$ & - & - & - & - \\
\hline X Sgr & $4.548 \pm 0.006$ & $3.652 \pm 0.006$ & $2.950 \pm 0.007$ & $2.635 \pm 0.007$ & $2.505 \pm 0.010$ & F08, W84 \\
\hline X Vul & $8.834 \pm 0.006$ & $7.198 \pm 0.020$ & $5.928 \pm 0.010$ & $5.433 \pm 0.008$ & $5.214 \pm 0.015$ & M11 \\
\hline XX Cen & $7.824 \pm 0.006$ & $6.743 \pm 0.006$ & $5.914 \pm 0.008$ & $5.541 \pm 0.006$ & $5.375 \pm 0.007$ & L92, W84 \\
\hline XX Mon & $11.914 \pm 0.007$ & $10.505 \pm 0.009$ & - & - & - & - \\
\hline XX Sgr & $8.869 \pm 0.006$ & $7.506 \pm 0.006$ & $6.412 \pm 0.033$ & $5.964 \pm 0.018$ & $5.799 \pm 0.022$ & W84 \\
\hline XX Vel & $10.676 \pm 0.006$ & $9.302 \pm 0.006$ & - & - & - & - \\
\hline XZ Car & $8.597 \pm 0.006$ & $7.248 \pm 0.006$ & - & - & - & - \\
\hline Y Aur & $9.809 \pm 0.044$ & - & $7.660 \pm 0.007$ & $7.291 \pm 0.008$ & $7.133 \pm 0.026$ & M11 \\
\hline Y Lac & $9.159 \pm 0.007$ & $8.308 \pm 0.026$ & $7.626 \pm 0.006$ & $7.316 \pm 0.006$ & $7.201 \pm 0.008$ & B97, M11 \\
\hline Y Oph & $6.148 \pm 0.006$ & $4.533 \pm 0.006$ & $3.349 \pm 0.006$ & $2.874 \pm 0.006$ & $2.662 \pm 0.008$ & W84, L92 \\
\hline Y Sct & - & - & $6.472 \pm 0.009$ & $5.897 \pm 0.011$ & $5.646 \pm 0.014$ & M11 \\
\hline Y Sgr & $5.739 \pm 0.006$ & $4.790 \pm 0.006$ & - & - & - & - \\
\hline YZ Aur & $10.346 \pm 0.009$ & - & $7.498 \pm 0.015$ & $6.905 \pm 0.011$ & $6.689 \pm 0.024$ & M11 \\
\hline YZ Car & $8.714 \pm 0.006$ & $7.438 \pm 0.006$ & - & - & - & - \\
\hline YZ Sgr & $7.351 \pm 0.006$ & $6.226 \pm 0.006$ & $5.379 \pm 0.007$ & $5.004 \pm 0.009$ & $4.861 \pm 0.010$ & M11, W84 \\
\hline Z Lac & $8.417 \pm 0.006$ & $7.198 \pm 0.043$ & $6.235 \pm 0.009$ & $5.811 \pm 0.006$ & $5.653 \pm 0.008$ & B97, M11 \\
\hline Z Sct & - & - & $6.962 \pm 0.017$ & $6.483 \pm 0.016$ & $6.282 \pm 0.017$ & M11 \\
\hline$\zeta \mathrm{Gem}$ & $3.889 \pm 0.006$ & $3.096 \pm 0.006$ & $2.538 \pm 0.006$ & $2.210 \pm 0.006$ & $2.096 \pm 0.006$ & F08 \\
\hline
\end{tabular}


Table 6. Sample of Large Magellanic Cloud Cepheids and their main parameters. The Cepheid names in the first column are of the form OGLE-LMC-CEP-XXXX. The uncertainties on $V$ and $I$ band mean magnitudes are 0.02 mag and the uncertainty on $E(B-V)$ values is $0.017 \mathrm{mag}$. The distances listed in column (5) are corrected for their position in the LMC by the equations provided in Sect. 3.2. Apparent magnitudes in this table are not corrected for the reddening. The full table is available as supplementary material.

\begin{tabular}{ccccccccccc}
\hline \hline Cepheid & $\begin{array}{c}\mathrm{P} \\
(\text { days })\end{array}$ & $\begin{array}{c}\alpha \\
(\mathrm{J} 2000)\end{array}$ & $\begin{array}{c}\delta \\
(\mathrm{J} 2000)\end{array}$ & $\begin{array}{c}d \\
(\mathrm{kpc})\end{array}$ & $\begin{array}{c}V \\
(\mathrm{mag})\end{array}$ & $\begin{array}{c}I \\
(\mathrm{mag})\end{array}$ & $\begin{array}{c}J \\
(\mathrm{mag})\end{array}$ & $\begin{array}{c}H \\
(\mathrm{mag})\end{array}$ & $\begin{array}{c}K_{S} \\
(\mathrm{mag})\end{array}$ & $\begin{array}{c}E(B-V) \\
(\mathrm{mag})\end{array}$ \\
\hline 0107 & 8.739 & 72.214 & -69.356 & $50.63 \pm 0.56$ & 14.761 & 13.947 & $13.332 \pm 0.018$ & $13.027 \pm 0.015$ & $12.941 \pm 0.014$ & 0.182 \\
0174 & 15.863 & 72.719 & -69.316 & $50.55 \pm 0.56$ & 14.739 & 13.666 & $12.876 \pm 0.022$ & $12.454 \pm 0.020$ & $12.312 \pm 0.019$ & 0.174 \\
0328 & 34.460 & 73.599 & -70.902 & $50.68 \pm 0.56$ & 13.124 & 12.088 & $11.460 \pm 0.031$ & $11.111 \pm 0.027$ & $11.001 \pm 0.024$ & 0.133 \\
0467 & 22.718 & 74.301 & -67.383 & $50.06 \pm 0.55$ & 13.704 & 12.775 & $12.113 \pm 0.033$ & $11.767 \pm 0.031$ & $11.668 \pm 0.029$ & 0.112 \\
0473 & 2.634 & 74.331 & -68.821 & $50.25 \pm 0.56$ & 16.335 & 15.590 & $15.093 \pm 0.082$ & $14.856 \pm 0.116$ & $14.641 \pm 0.114$ & 0.141 \\
0478 & 2.764 & 74.355 & -69.567 & $50.36 \pm 0.56$ & 16.160 & 15.471 & $14.961 \pm 0.057$ & $14.666 \pm 0.073$ & $14.624 \pm 0.076$ & 0.150 \\
0480 & 4.035 & 74.364 & -69.355 & $50.33 \pm 0.56$ & 16.865 & 15.779 & $15.107 \pm 0.049$ & $14.513 \pm 0.052$ & $14.515 \pm 0.080$ & 0.161 \\
0482 & 7.466 & 74.370 & -69.227 & $50.31 \pm 0.56$ & 15.655 & 14.661 & $13.977 \pm 0.081$ & $13.439 \pm 0.031$ & $13.315 \pm 0.030$ & 0.151 \\
0487 & 3.109 & 74.422 & -69.406 & $50.33 \pm 0.56$ & 16.221 & 15.469 & $15.002 \pm 0.124$ & $14.614 \pm 0.041$ & $14.488 \pm 0.090$ & 0.165 \\
0488 & 3.647 & 74.422 & -68.800 & $50.24 \pm 0.56$ & 16.535 & 15.608 & $14.921 \pm 0.037$ & $14.517 \pm 0.045$ & $14.451 \pm 0.066$ & 0.140 \\
0494 & 2.727 & 74.441 & -69.062 & $50.27 \pm 0.56$ & 16.973 & 16.013 & $15.441 \pm 0.087$ & $14.953 \pm 0.100$ & $14.658 \pm 0.091$ & 0.134 \\
0498 & 3.630 & 74.455 & -68.720 & $50.22 \pm 0.56$ & 15.914 & 15.154 & $14.617 \pm 0.071$ & $14.295 \pm 0.059$ & $14.286 \pm 0.061$ & 0.139 \\
0514 & 3.504 & 74.554 & -69.203 & $50.28 \pm 0.56$ & 16.276 & 15.458 & $14.879 \pm 0.063$ & $14.458 \pm 0.055$ & $14.381 \pm 0.068$ & 0.152 \\
0518 & 3.249 & 74.577 & -69.367 & $50.30 \pm 0.56$ & 16.550 & 15.589 & $14.988 \pm 0.044$ & $14.565 \pm 0.044$ & $14.543 \pm 0.046$ & 0.174 \\
0529 & 2.856 & 74.637 & -69.041 & $50.24 \pm 0.56$ & 16.603 & 15.777 & $15.168 \pm 0.087$ & $14.746 \pm 0.084$ & $14.698 \pm 0.087$ & 0.134 \\
0539 & 3.455 & 74.672 & -68.865 & $50.21 \pm 0.55$ & 16.064 & 15.320 & $14.758 \pm 0.056$ & $14.373 \pm 0.069$ & $14.299 \pm 0.054$ & 0.131 \\
0540 & 3.750 & 74.673 & -69.526 & $50.31 \pm 0.56$ & 15.841 & 15.069 & $14.574 \pm 0.061$ & $14.198 \pm 0.058$ & $14.188 \pm 0.056$ & 0.160 \\
$\ldots$ & $\ldots$ & $\ldots$ & $\ldots$ & $\ldots$ & $\ldots$ & $\ldots$ & $\ldots$ & $\ldots$ & $\ldots$ & $\ldots$ \\
\hline
\end{tabular}

Table 7. Sample of Small Magellanic Cloud Cepheids and their main parameters. The Cepheid names in the first column are of the form OGLE-SMC-CEP-XXXX. The uncertainties on $V$ and $I$ band mean magnitudes are 0.02 mag and the uncertainty on $E(B-V)$ values is $0.015 \mathrm{mag}$. The distances listed in column (5) are corrected for their position in the SMC by the equations provided in Sect. 3.3. Apparent magnitudes in this table are not corrected for the reddening. The full table is available as supplementary material.

\begin{tabular}{cccccccccc}
\hline \hline Cepheid & $\begin{array}{c}\mathrm{P} \\
(\text { days })\end{array}$ & $\begin{array}{c}\alpha \\
(\mathrm{J} 2000)\end{array}$ & $\begin{array}{c}\delta \\
(\mathrm{J} 2000)\end{array}$ & $\begin{array}{c}d \\
(\mathrm{kpc})\end{array}$ & $\begin{array}{c}V \\
(\mathrm{mag})\end{array}$ & $\begin{array}{c}I \\
(\mathrm{mag})\end{array}$ & $\begin{array}{c}J \\
(\mathrm{mag})\end{array}$ & $\begin{array}{c}K_{S} \\
(\mathrm{mag})\end{array}$ & $\begin{array}{c}E(B-V) \\
(\mathrm{mag})\end{array}$ \\
\hline 0443 & 4.037 & 10.525 & -73.061 & $63.36 \pm 0.95$ & 16.443 & 15.671 & $15.139 \pm 0.018$ & $14.742 \pm 0.006$ & 0.096 \\
0489 & 3.242 & 10.734 & -73.160 & $63.42 \pm 0.95$ & 16.493 & 15.822 & $15.096 \pm 0.006$ & $14.492 \pm 0.010$ & 0.101 \\
0494 & 4.758 & 10.742 & -73.335 & $63.72 \pm 0.96$ & 15.884 & 15.025 & $14.447 \pm 0.006$ & $14.030 \pm 0.004$ & 0.103 \\
0495 & 6.312 & 10.743 & -73.092 & $63.30 \pm 0.95$ & 16.038 & 15.217 & $14.604 \pm 0.012$ & $14.164 \pm 0.004$ & 0.097 \\
0499 & 6.229 & 10.751 & -73.304 & $63.66 \pm 0.96$ & 15.986 & 15.258 & $14.740 \pm 0.004$ & $14.374 \pm 0.006$ & 0.108 \\
0514 & 2.542 & 10.774 & -73.082 & $63.27 \pm 0.95$ & 16.842 & 16.145 & $15.687 \pm 0.010$ & $15.306 \pm 0.010$ & 0.097 \\
0518 & 15.773 & 10.802 & -73.326 & $63.67 \pm 0.96$ & 15.184 & 14.173 & $13.449 \pm 0.002$ & $12.948 \pm 0.004$ & 0.108 \\
0524 & 10.527 & 10.828 & -73.339 & $63.68 \pm 0.96$ & 15.383 & 14.536 & $13.934 \pm 0.008$ & $13.492 \pm 0.006$ & 0.105 \\
0533 & 3.909 & 10.859 & -73.254 & $63.52 \pm 0.95$ & 16.122 & 15.390 & $14.895 \pm 0.006$ & $14.489 \pm 0.010$ & 0.105 \\
0551 & 3.262 & 10.905 & -73.129 & $63.28 \pm 0.95$ & 16.473 & 15.807 & $15.302 \pm 0.006$ & $14.954 \pm 0.016$ & 0.101 \\
0570 & 10.883 & 10.947 & -73.241 & $63.45 \pm 0.95$ & 15.213 & 14.354 & $13.738 \pm 0.010$ & $13.294 \pm 0.004$ & 0.112 \\
0571 & 4.897 & 10.948 & -73.335 & $63.62 \pm 0.95$ & 15.872 & 15.178 & $14.662 \pm 0.006$ & $14.297 \pm 0.008$ & 0.104 \\
0576 & 14.426 & 10.963 & -73.333 & $63.61 \pm 0.95$ & 15.110 & 14.122 & $13.470 \pm 0.016$ & $12.923 \pm 0.018$ & 0.104 \\
0584 & 4.654 & 10.989 & -73.192 & $63.35 \pm 0.95$ & 16.058 & 15.296 & $14.777 \pm 0.006$ & $14.418 \pm 0.006$ & 0.111 \\
0596 & 3.072 & 11.013 & -73.277 & $63.48 \pm 0.95$ & 17.170 & 16.282 & $15.673 \pm 0.006$ & $15.184 \pm 0.012$ & 0.120 \\
$\ldots$ & $\ldots$ & $\ldots$ & $\ldots$ & $\ldots$ & $\ldots$ & $\ldots$ & $\ldots$ & & $\ldots$ \\
\hline
\end{tabular}

